\title{
Multisite phosphorylation by Cdk1 initiates delayed negative feedback to control mitotic transcription
}

Jonathan B. Asfaha ${ }^{1}$, Mihkel Örd², Mart Loog², and David O. Morgan ${ }^{1 *}$

1Department of Physiology, University of California, San Francisco, San Francisco CA 94143

${ }^{2}$ Institute of Technology, University of Tartu, Tartu, Estonia

*Correspondence: David.Morgan@ucsf.edu 


\section{Abstract}

Ordered phosphorylation of cyclin-dependent kinase (CDK) substrates leads to the sequential transcriptional activation and inhibition of hundreds of cell cycle-regulated genes. We find that Ndd1, an activator of genes required for mitotic progression, is both positively and negatively regulated by CDK activity. CDK activity initially stimulates Ndd1-dependent transcription as cells enter mitosis, but prolonged high CDK activity in a mitotic arrest inhibits transcription. The result is a time-delayed negative feedback circuit that generates a pulse of mitotic gene expression. Our results suggest that high CDK activity catalyzes the formation of multiple weak phosphodegrons on Ndd1, leading to its destabilization. Cyclin specificity and phosphorylation kinetics contribute to the timing of Ndd1 destruction. Failure to degrade Ndd1 in a mitotic arrest leads to elevated mitotic gene expression. We conclude that a combination of positive and negative Ndd1 regulation by CDKs governs the timing and magnitude of the mitotic transcriptional program.

\section{Introduction}

Progression through the steps of the cell division cycle requires the coordinated action of numerous regulatory programs. Cyclin-dependent kinases (CDKs) sit atop this regulatory hierarchy and phosphorylate hundreds of substrates in a specific order, thereby directing when and where each substrate executes its function ${ }^{1-3}$. In many 
cases, CDKs phosphorylate the same substrates at multiple sites to impart more complex forms of regulation ${ }^{4-7}$. We have little understanding, however, of how multisite phosphorylation generates complex regulatory behaviors.

Activation of the CDK catalytic subunit depends on association with transientlyexpressed cyclin subunits, which direct the formation of stage-specific cyclin-CDK complexes with distinct but overlapping functions ${ }^{3,8,9}$. CDKs generally phosphorylate substrates in regions of intrinsic disorder, with a clear preference for the minimal consensus $\left[\mathrm{S} / \mathrm{T}^{*}\right] \mathrm{P}$ or the full consensus $\left[\mathrm{S} / \mathrm{T}^{\star}\right] \mathrm{Px}[\mathrm{K} / \mathrm{R}]^{2}$. These sites are often found in clusters, resulting in the potential for complex patterns of multisite phosphorylation $2,10,11$.

Substrate phosphorylation is governed in part by interactions with the catalytic and regulatory subunits of cyclin-CDK complexes. The sequence context surrounding phosphorylation sites has a major impact on substrate affinity and catalytic rate ${ }^{12}$. As a result, the rate of phosphorylation, even by the same cyclin-CDK complex, varies over several orders of magnitude for different substrates and for different sites in the same substrate $^{1,3}$. In addition, many substrates contain cyclin-docking motifs, which interact with binding sites on a specific cyclin subunit to enhance phosphorylation at certain cellcycle stages $3,7,13-15$. Substrate interactions are also influenced by the Cks1 subunit of the cyclin-CDK complex, which interacts with specific phosphothreonine priming sites to promote phosphorylation at other sites ${ }^{5,16}$. These and other mechanisms result in wide variations in substrate affinity, which are expected to influence the timing of phosphorylation during the cell cycle. It seems likely that higher-affinity sites are 
phosphorylated more rapidly and earlier, while lower-affinity sites are modified at a slower rate and later.

In addition to binding determinants on substrates, variations in the intrinsic catalytic activities of cyclin-CDK complexes influence the timing of substrate phosphorylation. Overall CDK activity is low in G1 and rises to peak levels as cells progress into mitosis ${ }^{17}$. Strikingly, the rising activity of a single cyclin-CDK complex triggers the correct order of cell cycle events in the fission yeast Schizosaccharomyces pombe ${ }^{18}$. In the budding yeast Saccharomyces cerevisiae, different cyclins have different effects on the active site of the major CDK, Cdk1: early (G1/S) cyclins generate lower catalytic activity than later mitotic cyclins, resulting in an overall increase in general Cdk1 activity from G1 to mitosis ${ }^{19}$. This gradual rise in activity presumably leads to ordered substrate phosphorylation due to the variations in substrate affinity described above, perhaps together with variations in opposing phosphatase activities ${ }^{20}$.

In S. cerevisiae, ordered substrate phosphorylation by Cdk1 enables the sequential transcription of large numbers of genes during cell cycle progression ${ }^{21}$. Genes that are coordinately activated and repressed at the same cell cycle stage are grouped into transcriptional clusters, each of which generates proteins that enable progression into the next phase of the cell cycle 22 .

The major gene cluster controlling entry into mitosis is the CLB2 cluster, which includes the gene encoding the major mitotic cyclin, Clb222,23. The CLB2 transcriptional cluster is activated at the end of $S$ phase and encodes proteins required for progression through mitosis. Activation begins with the recruitment of Fkh2 to Mcm1 at CLB2 
cluster promoters ${ }^{24}$. The Fkh1 protein is also recruited to these promoters ${ }^{24}$.

Phosphorylation of C-terminal sites in Fkh2 by the S-phase Clb5-Cdk1 complex recruits the rate-limiting transcriptional co-activator $\mathrm{Ndd1}^{25}$. As the cell approaches mitosis, Ndd1 is phosphorylated at T319 by later cyclin-Cdk1 complexes, which promotes Ndd1 binding to the N-terminal FHA domain of Fkh226,27. FHA association, plus Cdc5mediated S85 phosphorylation on Ndd1, drives the activation of CLB2 cluster transcription ${ }^{26,28}$. Increased CLB2 expression leads to higher levels of Clb2-Cdk1 activity, which reinforces transcriptional activation by further phosphorylating Ndd1 in a positive feedback loop 25,29.

Surprisingly, although $\mathrm{Ndd1}$ is an essential protein that is required for transcription in mitosis, it is highly unstable in a long-term mitotic arrest ${ }^{30}$. Mutation of all 16 predicted CDK sites, or Cdk1 inhibition, is sufficient to stabilize the protein, suggesting that Cdk1 destabilizes Ndd1 in a mitotic arrest ${ }^{30}$. Ndd1 degradation in this arrest depends on the ubiquitin ligase SCF-Grr1 ${ }^{30}$.

SCF is a ubiquitin ligase that derives its substrate specificity from association with different F-box proteins, which typically interact with specific substrate sequence motifs called degrons ${ }^{31}$. Some F-box proteins adopt WD40 beta-propeller or arc-shaped leucine-rich-repeat (LRR) structures that bind degrons that are phosphorylated at one or more sites. Thus, kinases that create phosphodegrons can couple SCF-dependent protein degradation to cell cycle events.

The interaction between the F-box protein Cdc4 and its phosphodegron has been studied in considerable detail. Cdc4 contains a WD40 domain with a central binding site 
for the consensus phosphodegron $[\mathrm{I} / \mathrm{L} / \mathrm{P}][\mathrm{I} / \mathrm{L}][\mathrm{pS} / \mathrm{pT}] \mathrm{P}\langle\mathrm{R} / \mathrm{K} / \mathrm{Y}\rangle_{4}$ (where $\langle\mathrm{x}\rangle$ refers to disfavored residues) ${ }^{10}$. High-affinity Cdc4 phosphodegrons typically include a second phosphorylation site 2-4 residues downstream of the consensus site ${ }^{32}$. Some Cdc4 substrates, such as Eco1, require multiple kinases to create a diphosphodegron, enabling highly regulated and specific degradation ${ }^{4}$. Other Cdc4 targets, such as Sic1, contain two diphosphodegrons that are required for efficient degradation, suggesting that multiple phosphodegrons work together to promote substrate degradation ${ }^{14}$.

Some unstable proteins contain stretches of sequences rich in proline $(P)$, glutamate $(E)$, serine $(S)$, and threonine $(T)$ (called PEST sequences). PEST-containing substrates, such as Cln1/2 and Hof1, require phosphorylation at multiple sites inside and outside their PEST sequences to be degraded by SCF in a complex with the F-box protein Grr133-36. Recognition of phosphorylated substrates by Grr1 is mediated through a critical cationic surface on its LRR domain ${ }^{37}$. While some sequence features have been uncovered for Grr1-mediated degradation, the specific Grr1 phosphodegron sequence remains unknown. It appears, however, that phosphorylation at multiple sites is required for degradation of Grr1-specific substrates.

In the current work, we explored the mechanisms by which Cdk1 controls CLB2 cluster gene expression. We find that expression is governed by an antagonistic regulatory program: Cdk1 initiates transcriptional activation but also represses it as kinase activity rises to higher levels. Our evidence suggests that the accumulation of high Clb2-Cdk1 activity in a mitotic arrest leads to the formation of multiple weak phosphodegrons on Ndd1, resulting in Ndd1 degradation and reduced CLB2 
expression. Thus, transcription of the CLB2 gene cluster is controlled by a combination of rapid positive feedback and delayed negative feedback acting on the same regulatory protein, generating a pulse of $C L B 2$ gene cluster transcription in a mitotic arrest.

\section{Results}

\section{Mitotic gene expression declines when cells are arrested in mitosis}

We analyzed expression of $C L B 2$ transcriptional cluster genes by measuring mRNA levels with RT-qPCR. As in previous studies, we found that expression of genes in this cluster reaches peak levels in mitosis, 60 min after release from a G1 arrest (Figure 1a) ${ }^{22}$. Transcriptional activation is driven by M-phase Cdk1 activity, which reaches maximum levels in mitosis ${ }^{17,29,38}$. We therefore predicted that high CDK activity in a mitotic arrest would sustain continuous transcription of genes in the CLB2 transcriptional cluster. To test this possibility, we arrested cells in $\mathrm{G} 1$ and released them into nocodazole, a microtubule poison that activates the spindle assembly checkpoint and arrests cells in mitosis. Surprisingly, we found that upon entry into a mitotic arrest, expression of genes in the CLB2 transcriptional cluster decreased to between $50 \%$ and $20 \%$ of peak levels (Figure 1 b). Similar results were obtained when cells were arrested in mitosis by $C D C 20$ depletion, indicating that the effect was not due to spindle assembly checkpoint activation (Figure 1c).

Despite the reduction in transcript levels, Clb2 protein levels remained at a constant high level in a mitotic arrest, as seen in previous studies (Figure 1d) ${ }^{39-41}$. Clb2 
is thought to be very stable in a mitotic arrest, which would explain its high steady-state levels despite the decline in its mRNA transcript.

\section{Negative feedback reduces expression of genes in the CLB2 transcriptional}

\section{cluster in a mitotic arrest}

Cdk1-mediated positive feedback promotes CLB2 transcriptional activation in mitosis $^{25,29}$. We hypothesized that high mitotic Cdk1 activity might also suppress CLB2 transcription. We inhibited Cdk1 at various inhibitor concentrations in an analogsensitive Cdk1 mutant, cdk1-as1. Cdk1 in this strain bears an F88G mutation in the active site, making Cdk1 highly selective for the inhibitor 1-NM-PP1 ${ }^{42}$. Mild Cdk1 inhibition, at $50 \mathrm{nM}$ or $100 \mathrm{nM} 1-\mathrm{NM}-\mathrm{PP} 1$, delayed the reduction in CLB2 transcription (Figure 1e). Mild inhibition did not significantly affect the initial mitotic rise in CLB2 expression, suggesting that partial Cdk1 inhibition did not affect the positive feedback required for transcriptional activation. However, strong Cdk1 inhibition, at 250 nM or 500 nM 1-NM-PP1, caused a dramatic suppression of transcription, presumably through inhibition of positive feedback (Figure 1f). Together, these data demonstrate that strong Cdk1 inhibition inhibits positive feedback and transcriptional activation, while mild inhibition blocks negative feedback while leaving positive feedback less affected. A simple explanation for these results is that negative feedback requires higher levels of kinase activity and is therefore more readily inhibited by partial kinase inhibition. 
Ndd1 is a key positive regulator of the CLB2 transcriptional cluster and is regulated by Cdk1-mediated phosphorylation ${ }^{26}$. We therefore tested its role in the negative feedback we observed. Immunoblotting revealed that Ndd1 levels decreased as cells proceeded into a mitotic arrest from $\mathrm{G} 1$, and this decline could be alleviated by the addition of $100 \mathrm{nM}$ 1-NM-PP1 (Figure 2a, b).

To explore the possibility that Cdk1 destabilizes Ndd1, we measured the effect of Cdk1 inhibition on Ndd1 stability at various points after release from $\mathrm{G} 1$. We estimated Ndd1 half-life by measuring its decline after addition of cycloheximide at 60,90 , or 150 min after release from $\mathrm{G} 1$ into a mitotic arrest. To test the role of Cdk1 in destabilizing Ndd1, we also performed parallel experiments in the presence of strong Cdk1 inhibition. We found that Ndd1 degradation rate increased as cells proceeded into the mitotic arrest (Figure 2c; blue). Inhibition of Cdk1 with 500 nM 1-NM-PP1 prevented Ndd1 degradation at all time points (Figure 2c; red). These data suggest that Cdk1-dependent Ndd1 degradation rate increases gradually during a mitotic arrest, suggesting that prolonged high Cdk1 activity is required to promote degradation.

If Ndd1 stability is tied to cell cycle stage, then cyclin specificity may be an important determinant of Ndd1 destruction. The two major mitotic cyclins, Clb3 and Clb2, are thought to act in sequence as cells approach and progress into mitosis, so we tested their contributions to Ndd1 stability. Deletion of Clb3 had no effect, whereas deletion of Clb2 increased the stability of Ndd1 (Figure 2d). Thus, Ndd1 instability is likely to be coupled to rising Clb2-dependent Cdk1 activity during a prolonged mitotic arrest. This result raises the possibility that phosphorylation sites involved in 
destabilization are Clb2-specific, although they might simply be suboptimal Cdk1 sites if high Clb2-Cdk1 activity is required for degradation.

\section{Multiple phosphorylation sites are required for Ndd1 destruction}

To identify the regions of $\mathrm{Ndd} 1$ that are required for degradation, we first assessed the stability of Ndd1 truncation mutants. Deletion of the amino-terminal 115 amino acids stabilized Ndd1 (Figure 3a). Two minimal CDK consensus sites, S47 and T57, are found within this region. Consistent with the importance of these sites, an S47A substitution partially stabilized Ndd1, while a T57A substitution led to a greater level of stabilization (Figure 3b).

Mutation of all 16 CDK consensus sites in Ndd1 (the Ndd1-16A mutant) is known to stabilize the protein in a mitotic arrest ${ }^{30}$. We confirmed the stability of this mutant (Figure 3c) and found that adding back S47 and T57 to this mutant was not sufficient to induce degradation (Figure 3c). Thus, S47 and T57 are required but not sufficient for rapid Ndd1 degradation.

Ndd1 contains multiple predicted CDK sites that are conserved in budding yeasts (Figures 3a, 4a; Supplementary Figure 1). Because S47 and T57 are not sufficient to induce degradation, we searched for other sites that contribute to Ndd1 destabilization. We systematically added back clusters of predicted CDK sites to Ndd1-16A, starting at the amino terminus, and measured the stability of each mutant. Adding back predicted CDK sites up to and including T236 (the Ndd1-8A mutant) had minimal effects on 
degradation rate (Figure 4b). However, further addition of S254 and T265 (the Ndd1-6A mutant) led to a major decline in stability (Figure 4c). Addition of more predicted Cterminal CDK sites further destabilized the protein, but to a smaller degree (Figure 4c).

Given the importance of S254 and T265 in destabilizing Ndd1, we asked whether one or both sites are sufficient for degradation. We added back either S254 or T265 to Ndd1-8A. Addition of either site led to an intermediate level of destabilization that fell between those of Ndd1-8A and Ndd1-6A (Figure 5a). Thus, the two sites work together to induce destabilization. Interestingly, however, when S254 and T265 are both mutated to alanine in an otherwise wild-type protein, Ndd1 is not stabilized (Figure 5b). Furthermore, a version of Ndd1 that contains only S47, T57, S254, and T265 is stable (Figure 5c), showing that these four sites, despite their contributions in some contexts, are not sufficient for degradation. Finally, a mutant containing only T57, S254, and T265, plus all sites C-terminal of T265, was partially destabilized (Figure 5d), consistent with evidence in Figure 4c that sites C-terminal of T265 are sufficient to promote some degradation. Together, our studies of numerous site mutations suggest that multiple redundant sites collaborate to induce Ndd1 degradation.

\section{Different CDK sites in Ndd1 display varying cyclin specificity and intrinsic rates}

\section{of phosphorylation}

Our results indicate that Ndd1 degradation requires high Cdk1 activity that depends on Clb2 but not Clb3. Previous biochemical studies have demonstrated that Ndd1 is a 
substrate of both Clb3-Cdk1 and Clb2-Cdk1, with a preference for Clb3-Cdk1 19,43. Since Clb3 levels rise before those of Clb2, we reasoned that sites implicated in Ndd1 activation might be Clb3-specific, while sites involved in degradation might be Clb2specific $^{15}$. We tested the ability of purified Clb3- or Clb2-Cdk1 complexes to phosphorylate Ndd1 in vitro (Figure 6a), confirming a slight overall preference for Clb3Cdk1. We then used quantitative mass spectrometry to measure the ratio of detectable phosphorylated peptides between Clb2- and Clb3-dependent reactions (Figure 6b). We found that sites C-terminal of T277 display moderate Clb3 specificity. These sites include T319, which is required for Ndd1 activation ${ }^{26}$. Another Clb3-specific site, S370, is not a CDK consensus site but was nevertheless phosphorylated. In contrast, one site, T265, displayed significant Clb2 specificity.

While cyclin specificity may play a role in determining the timing of Ndd1 activation and degradation, it seems likely that the intrinsic rates of phosphorylation at different sites are also important factors. Using mass spectrometry, we measured phosphorylation at detectable sites after incubation of $1 \mu \mathrm{M}$ Ndd1 at either low initial substrate turnover (4 minutes phosphorylation with 400 pM Clb2-Cdk1) or high substrate turnover (40 minutes phosphorylation with 2 nM Clb2-Cdk1) (Figure 6c). We reasoned that most sites were close to saturation at the high kinase concentration.

Thus, the fraction of this maximal phosphorylation at a low (limiting) kinase concentration should provide an estimate of the relative intrinsic rate of phosphorylation at that site (Figure 6d). The results suggest that S254 is a preferred site for Clb2-Cdk1, 
whereas several other sites, including T265, were phosphorylated at a relatively low rate.

Phosphorylation at S47 and T57 was not measurable in these experiments because the peptide containing these sites was not detected in the mass spectrometer (Supplementary Figure 2). However, we could not detect significant phosphorylation when we analyzed a mutant protein in which T57 was the only consensus CDK site (Figure 6c, lane 4). Thus, despite its clear role in regulating Ndd1 stability in vivo, T57 appears to be a poor substrate for the kinase in vitro. Given that high Clb2-Cdk1 activity in a mitotic arrest is required for gradual Ndd1 degradation, it is perhaps not surprising that some of the key destabilizing sites are suboptimal.

Our results suggest that Ndd1 degradation is responsible for the decline in CLB2 gene expression during a mitotic arrest. We tested this possibility by analyzing CLB2 mRNA levels in a yeast strain carrying a stabilized Ndd1 mutant. Of the point mutants that stabilized Ndd1, we found that the T57A substitution alone provided clear stabilization without deleterious effects on Ndd1 activation. We therefore constructed a yeast strain in which NDD1 was replaced with a mutant gene encoding Ndd1-T57A. When this strain was released from a G1 arrest into nocodazole, Ndd1-T57A protein levels remained high (Figure 6e). This mutant did not affect the initial rate of increase in $C L B 2$ transcription or the timing of the peak of maximum transcription. However, CLB2 mRNA levels accumulated to higher levels in the mutant strain, suggesting that Ndd1 degradation helps control the amplitude of transcriptional activation (Figure 6f). 


\section{Discussion}

Expression of genes in the CLB2 transcriptional cluster is known to be stimulated by Cdk1 activity, suggesting that the mitotic rise in CLB2 expression depends, at least in part, on positive feedback ${ }^{25,27,29}$. Our work suggests that this system also contains negative feedback: Cdk1 suppresses CLB2 expression by catalyzing a complex series of phosphorylation events that destabilize Ndd1. Furthermore, our studies with a range of Cdk1 inhibitor concentrations suggest that the quantity of Cdk1 activity determines its effects. Low kinase activity is sufficient to stimulate CLB2 expression, whereas higher activity is required to repress expression. We propose that early Cdk1 activity at the CLB2 promoter stimulates the production of Clb2-Cdk1 activity as cells enter mitosis, after which accumulation of high Clb2-Cdk1 activity in mitosis leads to delayed negative feedback that inhibits transcription through Ndd1 degradation. This time-delayed negative feedback system generates a pulse of mitotic transcription in a mitotic arrest ${ }^{44}$.

The gradual rise in mitotic Cdk1 activity in a mitotic arrest leads to a gradual increase in the rate of degradation of Ndd1. Through this rheostatic stability control mechanism, the transcriptional output of Ndd1 is tuned by the time spent in a mitotic arrest. We speculate that coupling high Cdk1 activity to Ndd1 destabilization serves to constrain the impact of positive feedback in a prolonged mitotic state.

Although it is known that mitotic Clb3- and Clb2-Cdk1 complexes phosphorylate Ndd1 in vitro, it remains unclear how they regulate its activity ${ }^{19,43}$. Evidence from Kõivomägi et al. ${ }^{19}$ indicates that the catalytic efficiency of cyclin-Cdk1 complexes increases from $\mathrm{G} 1$ to $\mathrm{M}$ phase. We find that although Ndd1 appears to be a moderately 
Clb3-specific substrate in vitro, Ndd1 destabilization depends primarily on Clb2 (Figure 2d). We therefore suspect that accumulation of high catalytic efficiency Clb2-Cdk1 complexes is required to phosphorylate suboptimal sites on Ndd1, explaining the low Clb2-dependent phosphorylation rates we see in vitro.

We found that T57 is not detectably phosphorylated in vitro but has a clear role in destabilizing Ndd1 in vivo. Poor phosphorylation of this site in vitro likely reflects the need for very high levels of Clb2-Cdk1 activity in vivo for its modification. Similarly, the slow phosphorylation rate but increased Clb2 specificity at T265 suggest that this site requires high catalytic efficiency to be modified and destabilize Ndd1. Considering that Clb2-Cdk1 activity accumulates to high levels in a mitotic arrest, it is likely that several suboptimal sites (such as S47, T57, and T265) are only phosphorylated later in mitosis.

If later Clb2-dependent phosphorylation events destabilize Ndd1, then early phosphorylation events, catalyzed by Clb3-Cdk1, are likely to activate Ndd1. Indeed, previous studies suggest that $C L B 2$ transcription is significantly lower in the absence of $\mathrm{Clb3}^{29}$. This requirement for Clb3 presumably reflects its role in initiating Ndd1 activation before positive feedback is triggered by $\mathrm{Clb} 2$ and Cdc5. Other work has demonstrated that Cdk1-dependent phosphorylation of T319 on Ndd1 is critical for its promoter recruitment and thus for $C L B 2$ transcriptional activation ${ }^{26}$. Ndd1 promoter recruitment is therefore coupled to early mitotic cyclin activity. Our mass spectrometry studies revealed that T319 displays specificity for $\mathrm{Clb3}$ over $\mathrm{Clb} 2$, and previous studies have demonstrated that Ndd1 phosphorylation depends in part on the substrate-docking 
pocket of $\mathrm{Clb}^{43}$. Early mitotic phosphorylation of T319 could depend on a nearby Cterminal Clb3-docking motif.

Multiple sites in the C-terminal region of Ndd1 enhance the rate of Ndd1 degradation (Figure 4c, 5d). Some of these sites display moderate Clb3 specificity (Figure 6c), suggesting that early Clb3-dependent C-terminal phosphorylation contributes to Ndd1 degradation as the cell enters mitosis. However, although $\mathrm{Clb3}$ is abundant in nocodazole-arrested cells, our results argue that it is not required for Ndd1 degradation in those cells (Figure $2 \mathrm{~d})^{41}$. Phosphorylation of C-terminal sites by Clb2Cdk1 appears to be sufficient to promote degradation in a mitotic arrest.

Ndd1 contains an evenly distributed array of many predicted CDK sites. Our work suggests that several sites act redundantly to promote Ndd1 degradation. This concept became particularly apparent when the addition of S254 and T265 to Ndd1-8A induced faster degradation, whereas an S254A T265A double mutant was not more stable than wild-type Ndd1. In other words, S254 and T265 are sufficient to promote degradation in a sensitized mutant, but not required for degradation in wild-type Ndd1. Furthermore, when we went on to dissect the contribution of either S254 or T265 to the stability of Ndd1-6A, we observed that each site partially destabilized Ndd1. The simplest explanation for these and our other results is that degradation depends on a combination of multiple suboptimal phosphorylation sites.

Some CDK substrates are phosphorylated at multiple sites in a semi-processive manner, due to the ability of the associated Cks1 subunit to interact with prephosphorylated 'priming' sites ${ }^{5}$. However, few CDK sites in Ndd1 display greatly 
enhanced phosphorylation in the presence of $\mathrm{Cks} 1^{5}$, suggesting that most phosphorylation is distributive. If degradation requires simultaneous phosphorylation at multiple sites, then distributive phosphorylation can provide a system in which degradation is achieved only at high kinase thresholds, as we propose for Ndd1.

Cdk1-dependent Ndd1 degradation is likely to result from the binding of phosphodegrons to the Grr1 F-box subunit of the ubiquitin ligase SCF${ }^{30}$. Although multiple substrates of Grr1 have been discovered, its mechanisms of substrate interaction are not defined. Structural modeling and mutational analyses have provided important clues ${ }^{37}$. The central LRR domain forms an arc-like structure comprised of an outer arc of alpha helices that positions an inner surface of beta-sheets for substrate recognition. These beta sheets contain a high density of positively-charged residues that are necessary for recognizing phosphosites on Grr1-specific substrates. Mutations that span the inner cationic surface of Grr1 disrupt substrate binding, suggesting that Grr1 contains multiple phosphate-binding sites ${ }^{37}$. Regions that destabilize Grr1 substrates, as seen in Cln2 and Hof1, are enriched in clusters of phosphorylation sites that collectively destabilize these proteins ${ }^{33-36}$. Thus, Grr1 might recognize clusters of phosphorylation, as opposed to the relatively well-defined diphosphodegron recognized by the F-box protein Cdc4.

Curiously, many of the CDK sites in Ndd1 are grouped into pairs of sites 10-12 residues apart (Supplementary Figure 1). We found that the most N-terminal pair of predicted CDK sites, S47 and T57, is necessary but not sufficient for rapid degradation, while another closely-spaced pair, S254 and T265, also contribute. Additional 
phosphosites are needed to induce rapid degradation. It is conceivable that T57 and S47, or S254 and T265, together encode specificity for Grr1 but do not bind with sufficient affinity to maintain a stable interaction. For example, the avidity imparted by additional weakly-interacting phosphosites binding to the cationic surface of Grr1 might be required for stable association and degradation.

When we mutated the key destabilizing site at T57 to alanine, Ndd1 was greatly stabilized in vivo and transcriptional output accumulated to higher levels following a G1 release. However, despite the dramatic stabilization of $\mathrm{Ndd1}$, attenuated negative feedback was still observed. One potential explanation is that Ndd1-T57A is still degraded but at a significantly lower rate compared to Ndd1-WT. That is, the accumulation of many weak phosphodegrons over an extended period of time in a mitotic arrest may be sufficient to induce negative feedback, albeit at a slower rate. Alternatively, negative feedback might operate in part through mechanisms that do not depend on Ndd1 degradation.

Multisite phosphorylation mechanisms control a myriad of regulatory programs. Interestingly, the gradual accumulation of phosphorylation sites on Ndd1 resembles the multisite phosphorylation mechanism that activates the Anaphase-Promoting Complex or cyclosome (APC/C $)^{45-47}$. To be activated in mitosis, phosphorylation of several Cdk1 sites on the APC/C requires high mitotic kinase activity. As in Ndd1, these suboptimal phosphorylation sites on the APC/C are difficult to phosphorylate in vitro. Multisite phosphorylation mechanisms like those that regulate Ndd1 and the APC/C serve as biochemical control points to generate the correct timing of mitotic events. 


\section{Methods}

\section{General methods}

All strains are derivatives of W303a and listed in Supplementary Table 1. All strains were constructed using PCR- and/or restriction digest-based homologous recombination. Cells were grown in Yeast Extract Peptone (YEP) containing a combination of Raffinose, Galactose, Dextrose, or a combination thereof. For all experiments, cells were grown at $30^{\circ} \mathrm{C}$ and progressed through a minimum of two doubling times in log phase before any experiments were performed. For all time courses, cultures reached an $\mathrm{OD}_{595}$ between 0.15 and 0.3 before synchronization. Cells were synchronized in $\mathrm{G} 1 \mathrm{using} 1.5 \mu \mathrm{g} / \mathrm{ml} \mathrm{a}$-factor for $3 \mathrm{~h}$ at $30^{\circ} \mathrm{C}$ and released by washing three times in fresh media. Cells were arrested in mitosis by addition of 15 $\mu \mathrm{g} / \mathrm{ml}$ nocodazole for $3 \mathrm{~h}$ at $30^{\circ} \mathrm{C}$.

Ndd1 stability was analyzed in yeast strains expressing C-terminally Myc-tagged Ndd1 under the control of the GAL1-10 promoter. NDD1 expression was induced during mitotic synchronization by the addition of galactose to a final concentration of $2 \%$ in YEP-Raffinose. Cycloheximide (CHX) was added to a final concentration of $250 \mu \mathrm{g} / \mathrm{ml}$ to halt translation, and $1 \mathrm{ml}$ samples were harvested every $10 \mathrm{~min}$ to measure Ndd1 levels by Western blotting.

For Western blotting, yeast lysates were prepared by bead-beating cells in urea lysis buffer. Lysates were separated using SDS-PAGE and transferred in Tris/Glycine Buffer to a 0.45 micron Nitrocellulose membrane (GE-Healthcare Life Sciences). Blots 
were probed with the following antibodies diluted 1:5000 in TBS-T containing 5\% nonfat dry milk: mouse anti-Myc (Cell Signaling Technology Cat\# 2276, RRID:AB_331783), Rabbit anti-HA (Cell Signaling Technology Cat\# 3724), or Rabbit anti-Mouse IgG (H+L) Alexa Fluor 680 (Thermo Fisher Scientific, Cat\# A-21065, RRID AB_2535728). The following antibody was diluted 1:1500 in TBS-T containing 5\% nonfat dry milk: GAPDH Monoclonal Antibody (Thermo Fisher Scientific, Cat\# MA5-15738, RRID AB_10977387). Blots were imaged on an Odyssey Fc imager (LI-COR) at $700 \mathrm{~nm}$ for $10 \mathrm{~min}$.

For measurements of gene expression, total RNA was prepared by hot-acid phenol chloroform extraction. $5 \%$ of total RNA was DNase-treated in $50 \mu$ l reactions with TRUBO DNase (Thermo Fisher Scientific, Cat\# AM1907) for 30 min at $37^{\circ} \mathrm{C} .1 \mu \mathrm{l}$ was added to a $9 \mu$ L Luna ${ }^{\circledR}$ Universal One-Step RT-qPCR reaction (NEB, Cat\# E3005L). RT-qPCR was carried out to manufacturer specifications in a CFX96 Touch Real Time PCR Machine (Bio-Rad).

\section{Analysis of Ndd1 phosphorylation}

6xHis-tagged Ndd1 was expressed from pET28a in E. coli BL21RP cells at $30^{\circ} \mathrm{C}$ using 0.3 mM IPTG. 6xHis-Ndd1 was purified using immobilized nickel affinity chromatography and eluted with imidazole. Clb3- and Clb2-Cdk1 complexes were purified from $S$. cerevisiae lysates using TAP-tagged cyclins as described previously ${ }^{1,48}$. Cks1 was expressed in E. coli BL21RP and purified as described ${ }^{49}$. 
Kinase reactions were carried out at room temperature in $50 \mathrm{mM}$ Hepes- $\mathrm{KOH}$, $\mathrm{pH}$ 7.4, $150 \mathrm{mM} \mathrm{NaCl}, 5 \mathrm{mM} \mathrm{MgCl}$, $20 \mathrm{mM}$ imidazole, 2\% glycerol, $0.2 \mathrm{mg} / \mathrm{ml} \mathrm{BSA}$, and $500 \mu \mathrm{M}$ ATP. The concentration of $\mathrm{Ndd} 1$ was $1 \mu \mathrm{M}$ and of Cks1 was $500 \mathrm{nM}$. To quantitatively compare the phosphorylation of Ndd1 at different sites in different conditions, the reactions were supplemented with either normal isotopic ATP ([160]ATP) or heavy ATP ([180]ATP) (Cambridge Isotope Laboratories). For comparison of phosphorylation by Clb2- and Clb3-Cdk1, 0.4 nM Cdk1 complex was used and the reactions were stopped at $20 \mathrm{~min}$. To compare Ndd1 phosphorylation at low initial substrate turnover and high turnover, Ndd1 was phosphorylated with $0.4 \mathrm{nM}$ Clb2-Cdk1 for 4 min for low turnover, or $2 \mathrm{nM} \mathrm{Clb2-Cdk1} \mathrm{for} 40$ min for high turnover. The reactions were stopped with SDS loading buffer, and aliquots from different reactions were mixed together in a 1:1 ratio and analyzed by SDS-PAGE. The gel was stained with Colloidal Coomassie G-250 and the Ndd1 band was excised. In-gel digestion was performed using trypsin/P (20 ng/ $\mu \mathrm{l})$, and peptides were purified using C18 StageTips. Peptides were separated by an Agilent 1200 series nanoflow system (Agilent Technologies) and analyzed using an LTQ Orbitrap classic mass spectrometer (Thermo Electron) equipped with a nanoelectrospray ion source (Proxeon). Mascot 2.3 (Matrix Science) was used to identify the peptides. Two independent experiments were performed. 


\section{Acknowledgements}

We thank members of the Morgan laboratory for discussions and comments on the manuscript. This work was supported by an HHMI Gilliam Graduate Fellowship (to J.B.A), and a grant from the National Institute of General Medical Sciences (R35GM118053, to D.O.M.).

\section{Author contributions}

J.B.A. conceived the project, performed experiments and analyzed results, with guidance from D.O.M. M.Ö. and M.L. performed mass spectrometry analysis and interpretation. J.B.A. wrote the paper with assistance from all other authors.

\section{Competing interests}

The authors declare no competing interests. 


\section{References}

1. Ubersax, J. A. et al. Targets of the cyclin-dependent kinase Cdk1. Nature 425, 859-864 (2003).

2. Holt, L. J. et al. Global analysis of Cdk1 substrate phosphorylation sites provides insights into evolution. Science 325, 1682-1686 (2009).

3. Loog, M. \& Morgan, D. O. Cyclin specificity in the phosphorylation of cyclindependent kinase substrates. Nature 434, 104-108 (2005).

4. Lyons, N. A., Fonslow, B. R., Diedrich, J. K., Yates, J. R. \& Morgan, D. O.

Sequential primed kinases create a damage-responsive phosphodegron on Eco1. Nat Struct Mol Biol 20, 194-201 (2013).

5. Kõivomägi, M. et al. Multisite phosphorylation networks as signal processors for Cdk1. Nat Struct Mol Biol 20, 1415-1424 (2013).

6. Valk, E. et al. Multistep phosphorylation systems: tunable components of biological signaling circuits. Molecular Biology of the Cell 25, 3456-3460 (2014).

7. Örd, M., Venta, R., Möll, K., Valk, E. \& Loog, M. Cyclin-Specific Docking Mechanisms Reveal the Complexity of M-CDK Function in the Cell Cycle. Molecular Cell 75, 76-89 (2019).

8. Morgan, D. O. Cyclin-dependent kinases: engines, clocks, and microprocessors. Annu Rev Cell Dev Biol 13, 261-291 (1997).

9. Bloom, J. \& Cross, F. R. Multiple levels of cyclin specificity in cell-cycle control. Nat Rev Mol Cell Biol 8, 149-160 (2007).

10. Nash, P. et al. Multisite phosphorylation of a CDK inhibitor sets a threshold for the 
onset of DNA replication. Nature 414, 514-521 (2001).

11. Moses, A. M., Hériché, J.-K. \& Durbin, R. Clustering of phosphorylation site recognition motifs can be exploited to predict the targets of cyclin-dependent kinase. Genome Biol. 8, R23-14 (2007).

12. Holmes, J. K. \& Solomon, M. J. A predictive scale for evaluating cyclin-dependent kinase substrates. A comparison of p34cdc2 and p33cdk2. J. Biol. Chem. 271, 25240-25246 (1996).

13. Bhaduri, S. \& Pryciak, P. M. Cyclin-specific docking motifs promote phosphorylation of yeast signaling proteins by G1/S Cdk complexes. Curr. Biol. 21, 1615-1623 (2011).

14. Kõivomägi, M. et al. Cascades of multisite phosphorylation control Sic1 destruction at the onset of S phase. Nature 480, 128-131 (2011).

15. Örd, M. et al. Multisite phosphorylation code of CDK. Nat Struct Mol Biol 26, 649658 (2019).

16. McGrath, D. A. et al. Cks confers specificity to phosphorylation-dependent CDK signaling pathways. Nat Struct Mol Biol 20, 1407-1414 (2013).

17. Swaffer, M. P., Jones, A. W., Flynn, H. R., Snijders, A. P. \& Nurse, P. CDK Substrate Phosphorylation and Ordering the Cell Cycle. Cell 167, 1750-1761.e16 (2016).

18. Coudreuse, D. \& Nurse, P. Driving the cell cycle with a minimal CDK control network. Nature 468, 1074-1079 (2010).

19. Kõivomägi, M. et al. Dynamics of Cdk1 substrate specificity during the cell cycle. 
Molecular Cell 42, 610-623 (2011).

20. Godfrey, M. et al. PP2ACdc55 Phosphatase Imposes Ordered Cell-Cycle Phosphorylation by Opposing Threonine Phosphorylation. Molecular Cell 65, 393-402 (2017).

21. Wittenberg, C. \& Reed, S. I. Cell cycle-dependent transcription in yeast: promoters, transcription factors, and transcriptomes. Oncogene 24, 2746-2755 (2005).

22. Spellman, P. T. et al. Comprehensive identification of cell cycle-regulated genes of the yeast Saccharomyces cerevisiae by microarray hybridization. Molecular Biology of the Cell 9, 3273-3297 (1998).

23. Futcher, B. et al. Two yeast forkhead genes regulate the cell cycle and pseudohyphal growth. Nature 406, 90-94 (2000).

24. Kumar, R., Reynolds, D. M., Shevchenko, A., Goldstone, S. D. \& Dalton, S. Forkhead transcription factors, Fkh1p and Fkh2p, collaborate with Mcm1p to control transcription required for M-phase. Curr. Biol. 10, 896-906 (2000).

25. Pic-Taylor, A., Darieva, Z., Morgan, B. A. \& Sharrocks, A. D. Regulation of cell cycle-specific gene expression through cyclin-dependent kinase-mediated phosphorylation of the forkhead transcription factor Fkh2p. Molecular and Cellular Biology 24, 10036-10046 (2004).

26. Reynolds, D. et al. Recruitment of Thr 319-phosphorylated Ndd1p to the FHA domain of Fkh2p requires Clb kinase activity: a mechanism for CLB cluster gene activation. Genes Dev. 17, 1789-1802 (2003). 
27. Darieva, Z. et al. Cell cycle-regulated transcription through the FHA domain of Fkh2p and the coactivator Ndd1p. Curr. Biol. 13, 1740-1745 (2003).

28. Darieva, Z. et al. Polo kinase controls cell-cycle-dependent transcription by targeting a coactivator protein. Nature 444, 494-498 (2006).

29. Linke, C. et al. A Clb/Cdk1-mediated regulation of Fkh2 synchronizes CLB expression in the budding yeast cell cycle. NPJ Syst Biol App/ 3, 7-12 (2017).

30. Edenberg, E. R., Mark, K. G. \& Toczyski, D. P. Ndd1 turnover by SCF(Grr1) is inhibited by the DNA damage checkpoint in Saccharomyces cerevisiae. PLoS Genet. 11, e1005162 (2015).

31. Cardozo, T. \& Pagano, M. The SCF ubiquitin ligase: insights into a molecular machine. Nat Rev Mol Cell Biol 5, 739-751 (2004).

32. Bao, M. Z., Shock, T. R. \& Madhani, H. D. Multisite phosphorylation of the Saccharomyces cerevisiae filamentous growth regulator Tec1 is required for its recognition by the E3 ubiquitin ligase adaptor Cdc4 and its subsequent destruction in vivo. Eukaryot Cell 9, 31-36 (2010).

33. Salama, S. R., Hendricks, K. B. \& Thorner, J. G1 cyclin degradation: the PEST motif of yeast CIn2 is necessary, but not sufficient, for rapid protein turnover. Molecular and Cellular Biology 14, 7953-7966 (1994).

34. Quilis, I. \& Igual, J. C. A comparative study of the degradation of yeast cyclins CIn1 and CIn2. FEBS Open Bio 7, 74-87 (2017).

35. Berset, C. et al. Transferable domain in the $\mathrm{G}(1)$ cyclin Cln2 sufficient to switch degradation of Sic1 from the E3 ubiquitin ligase SCF(Cdc4) to SCF(Grr1). 
Molecular and Cellular Biology 22, 4463-4476 (2002).

36. Blondel, M. et al. Degradation of Hof1 by SCF(Grr1) is important for actomyosin contraction during cytokinesis in yeast. EMBO J 24, 1440-1452 (2005).

37. Hsiung, Y. G. et al. F-box protein Grr1 interacts with phosphorylated targets via the cationic surface of its leucine-rich repeat. Molecular and Cellular Biology 21, 2506-2520 (2001).

38. Surana, U. et al. Destruction of the CDC28/CLB mitotic kinase is not required for the metaphase to anaphase transition in budding yeast. EMBO J 12, 1969-1978 (1993).

39. Palframan, W. J., Meehl, J. B., Jaspersen, S. L., Winey, M. \& Murray, A. W. Anaphase inactivation of the spindle checkpoint. Science 313, 680-684 (2006).

40. Vernieri, C., Chiroli, E., Francia, V., Gross, F. \& Ciliberto, A. Adaptation to the spindle checkpoint is regulated by the interplay between Cdc28/Clbs and PP2ACdc55. The Journal of Cell Biology 202, 765-778 (2013).

41. Alexandru, G., Zachariae, W., Schleiffer, A. \& Nasmyth, K. Sister chromatid separation and chromosome re-duplication are regulated by different mechanisms in response to spindle damage. EMBO J 18, 2707-2721 (1999).

42. Bishop, A. C. et al. A chemical switch for inhibitor-sensitive alleles of any protein kinase. Nature 407, 395-401 (2000).

43. Örd, M. et al. Proline-Rich Motifs Control G2-CDK Target Phosphorylation and Priming an Anchoring Protein for Polo Kinase Localization. Cell Rep 31, 107757 (2020). 
44. Brandman, O. \& Meyer, T. Feedback loops shape cellular signals in space and time. Science 322, 390-395 (2008).

45. Rudner, A. D. \& Murray, A. W. Phosphorylation by Cdc28 activates the Cdc20dependent activity of the anaphase-promoting complex. The Journal of Cell Biology 149, 1377-1390 (2000).

46. Fujimitsu, K., Grimaldi, M. \& Yamano, H. Cyclin-dependent kinase 1-dependent activation of APC/C ubiquitin ligase. Science 352, 1121-1124 (2016).

47. Zhang, S. et al. Molecular mechanism of APC/C activation by mitotic phosphorylation. Nature 533, 260-264 (2016).

48. Puig, O. et al. The tandem affinity purification (TAP) method: a general procedure of protein complex purification. Methods 24, 218-229 (2001).

49. Reynard, G. J., Reynolds, W., Verma, R. \& Deshaies, R. J. Cks1 is required for $\mathrm{G}(1)$ cyclin-cyclin-dependent kinase activity in budding yeast. Molecular and Cellular Biology 20, 5858-5864 (2000). 


\section{Figure 1. Decline in CLB2 cluster gene expression in a mitotic arrest depends on}

\section{Cdk1 activity}

(a) Cells were released from a G1 arrest and harvested at the indicated times for analysis of gene expression by RT-qPCR. Data points indicate mean +/-S.E.M. in 3 independent experiments. (b) Gene expression was measured in cells released from G1 arrest into nocodazole (Noc) to induce a mitotic arrest. Data points indicate mean +/S.E.M. in 3 independent experiments. (c) Cells carrying an endogenous replacement of the $C D C 20$ promoter with a galactose-inducible promoter were released from a $\mathrm{G} 1$ arrest into dextrose to induce a mitotic arrest. CLB2 expression was measured at the indicated times by RT-qPCR. (d) Cells were released from G1 into nocodazole. Expression of 3xHA-tagged Clb2 was analyzed by immunoblot at the indicated times, with a GAPDH immunoblot serving as a loading control. (e \& f) Cells carrying analogsensitive Cdk1 (cdk1-as1) were released from G1 arrest into media containing the indicated concentrations of 1-NM-PP1, plus nocodazole. CLB2 gene expression was measured at the indicated times by RT-qPCR. Data points indicate mean +/-S.E.M. in 3 independent experiments.

Figure 2. Ndd1 destabilization requires Clb2-Cdk1 activity

(a) Cells were released from a G1 arrest into nocodazole. Myc-tagged Ndd1 and GAPDH expression were monitored by immunoblot at the indicated times. (b) cdk1-as 1 cells were released from a G1 arrest into nocodazole in the absence (left) or presence 
(right) of $100 \mathrm{nM}$ 1-NM-PP1. Myc-tagged Ndd1 and GAPDH expression were monitored by immunoblot at the indicated times. (c) Parallel cultures of cdk1-as1 cells were released from a G1 arrest into nocodazole, in the presence or absence of $500 \mathrm{nM} 1$ NM-PP1. Cycloheximide (CHX) was added after 60, 90, or 150 min. Samples were harvested after 0, 10, 20, or 30 min in $\mathrm{CHX}$. Myc-tagged Ndd1 levels were analyzed by immunoblot and quantified. Data points indicate mean +/-S.E.M. in 2 independent experiments. Estimated half-lives are summarized at lower right. (d) Cells expressing Ndd1-Myc under the control of the GAL promoter were grown in galactose and arrested in mitosis with nocodazole. $\mathrm{CHX}$ was added, and Ndd1-Myc and GAPDH were analyzed by immunoblotting at the indicated times.

Figure 3. Predicted N-terminal CDK sites are necessary but not sufficient for Ndd1 destabilization

(a) Left: Depiction of Ndd1 and an Ndd1 mutant lacking the first 115 amino acids (Ndd1$\Delta 115)$, with 16 consensus CDK sites marked (serine, red; threonine, orange). Right: Cells carrying galactose-inducible wild-type (WT) Ndd1 or Ndd1- $\Delta 115$ were grown in galactose and arrested in mitosis with nocodazole. CHX was added, and Ndd1-Myc and GAPDH were analyzed by immunoblotting at the indicated times. (b) Cells carrying Ndd1 (WT), Ndd1-S47A, or Ndd1-T57A were treated as in panel a. Left: Representative immunoblots of Ndd1-Myc and GAPDH. Right: Quantification of immunoblots from 3 independent experiments (mean +/- S.E.M.). (c) Cells carrying Ndd1-16A or Ndd1 containing only S47 and T57 (Ndd1-14A) were treated as in panel a. Left: 
Representative immunoblots of Ndd1-Myc and GAPDH. Right: Quantification of immunoblots from 3 independent experiments (mean +/- S.E.M.).

\section{Figure 4. Multiple predicted CDK sites promote Ndd1 destabilization}

(a) Diagram of predicted CDK sites in Ndd1, showing the sites mutated to alanine in the Ndd1 mutants analyzed below. (b) Cells carrying galactose-inducible Ndd1 alanine mutants $12 \mathrm{~A}, 10 \mathrm{~A}$, or $8 \mathrm{~A}$ (colors indicated in panel a) were grown in galactose and arrested in mitosis with nocodazole. $\mathrm{CHX}$ was added, and Ndd1-Myc was quantified by immunoblotting at the indicated times. Data points indicate mean +/-S.E.M. in 3 independent experiments. Dashed lines indicate data for Ndd1-16A and Ndd1-14A from Figure 3c. (c) Cells carrying galactose-inducible Ndd1 alanine mutants $6 \mathrm{~A}, 5 \mathrm{~A}$, or $3 \mathrm{~A}$ were treated and analyzed as in panel b. Data points indicate mean +/-S.E.M. in 3 independent experiments. Dashed line indicates data for WT Ndd1 from Figure 3b.

Figure 5. S254 and T265 require additional CDK sites to destabilize Ndd1

(a) Cells were constructed in which galactose-inducible Ndd1 carried alanine mutations at the C-terminal six CDK consensus sites (Ndd1-6A; see Figure 3a) plus a mutation of either S254 or T265 to alanine. Cells were grown in galactose and arrested in mitosis with nocodazole. CHX was added, and Ndd1-Myc was quantified by immunoblotting at the indicated times. Top: Quantification of immunoblots from 3 independent experiments (mean +/- S.E.M.). Dashed lines indicate data for Ndd1-8A from Figure 4b 
and Ndd1-6A from Figure 4c. Bottom: Representative immunoblots of Ndd1-Myc and GAPDH. (b) Cells carrying galactose-inducible Ndd1-S254, T265A were treated and analyzed as in panel a. Top: Quantification of immunoblots from 3 independent experiments (mean +/- S.E.M.). Dashed line indicates data for WT Ndd1 from Figure 4c. Bottom: Representative immunoblots of Ndd1-Myc and GAPDH. (c) Cells were constructed in which galactose-inducible Ndd1 carries alanine mutations at all CDK consensus sites except S47, T57, S254, and T265. Cells were treated and analyzed as in panel a. Top: Quantification of immunoblots from 3 independent experiments (mean +/- S.E.M.). Dashed line indicates data for WT Ndd1 from Figure 4c. Bottom:

Representative immunoblots of Ndd1-Myc and GAPDH. (d) Cells carrying galactoseinducible Ndd1 containing only T57, S254, T265, T277, T319, T332, S357, S409, and T411 (Ndd1-C1) were treated and analyzed as in panel a. Top: Quantification of immunoblots from 3 independent experiments (mean +/- S.E.M.). Dashed lines indicate data for Ndd1-16A and Ndd1-6A from Figure 3b and 3c, respectively. Bottom: Representative immunoblots of Ndd1-Myc and GAPDH.

Figure 6. Multiple CDK sites exhibit varying cyclin specificities and phosphorylation rates

(a) 400 pM purified Clb3-Cdk1 or Clb2-Cdk1 was incubated in a kinase reaction with 1 $\mu \mathrm{M} \mathrm{Ndd1}$ and ${ }^{32} \mathrm{P}-\gamma$-ATP. After 20 min, reaction products were analyzed by SDS-PAGE and autoradiography. (b) $400 \mathrm{pM}$ Clb3- or Clb2-Cdk1 were incubated with $1 \mu \mathrm{M}$ Ndd1 in kinase reactions supplemented with either light isotopic ATP ([16 O]ATP) or heavy 
isotopic ATP ([180]ATP), respectively, to quantitatively compare phosphorylation rates with the two kinases. Tryptic peptides containing the indicated phosphorylation sites were measured using mass spectrometry, and the ratio of phosphorylation by Clb2Cdk1 to Clb3-Cdk1 is indicated. Data points (mean +/-S.D.) are from 2 independent experiments. (c) Wild-type (WT) Ndd1 was phosphorylated with radiolabeled ATP in kinase reactions with 400 pM Clb2-Cdk1 for 4 min (low, lane 1) or 2 nM Clb2-Cdk1 for 40 min (high, lanes 2-4). In lanes 3 and 4, the high kinase concentration was used with mutant Ndd1 lacking all predicted CDK sites (Ndd1-16A) or a version lacking all predicted CDK sites except T57 (Ndd1-15A). Reaction products were analyzed by SDSPAGE and autoradiography. (d) Reactions were carried out with low or high Clb2-Cdk1 concentrations as in panel c, supplemented with heavy or light ATP as in panel b. The rate of phosphorylation at the indicated sites is expressed as a ratio of the amount of phosphorylation in the low kinase reaction to the amount of phosphorylation in the high kinase reaction. Data points (mean +/-S.D.) are from 2 independent experiments. (e) Cells carrying Myc-tagged WT Ndd1 or Ndd1-T57A were released from a G1 arrest into nocodazole to induce a mitotic arrest. Myc-tagged Ndd1 and GAPDH levels were analyzed by immunoblotting at the indicated times. (f) Cells carrying WT Ndd1 or Ndd1T57A were released from G1 arrest into nocodazole. Samples were harvested at the indicated times for analysis of CLB2 gene expression by RT-qPCR. Dashed line indicates data for WT from Figure 1b. Data points indicate mean +/-S.E.M. in 3 independent experiments. 
a bioRxiv preprint doi: https://doi.org/10.1101/2021.02.14.431158; this Wersion posted February 14, 2021. The copyright holder for this preprint

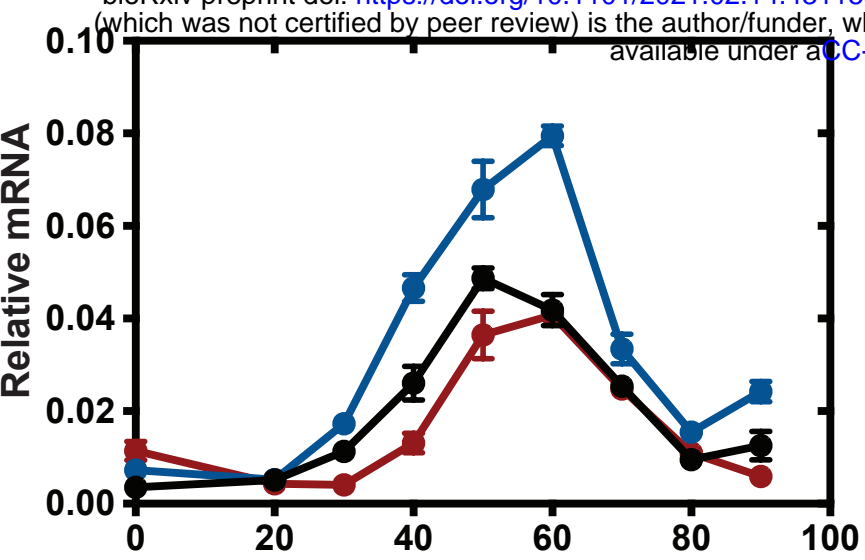

Time after G1 Release (min)

- CDC20 - CDC5 - CLB2

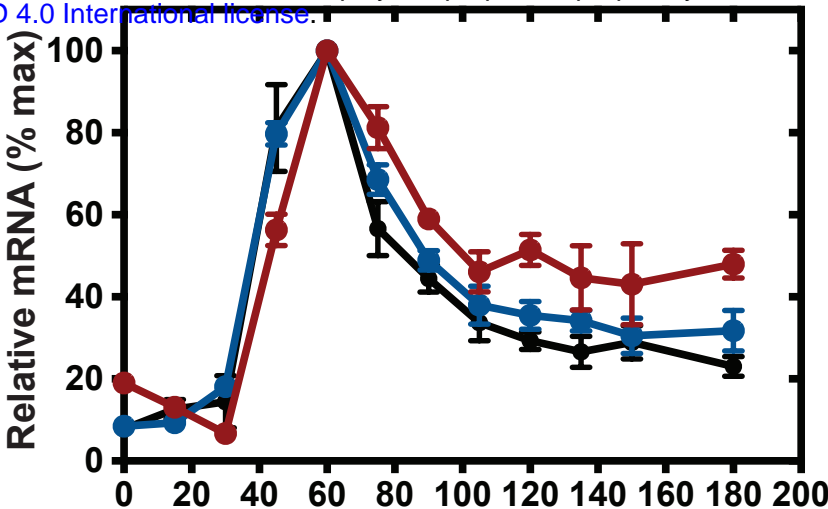

Time after $\mathbf{G} 1$ release into Noc ( $\mathrm{min})$

CLB2

C

d

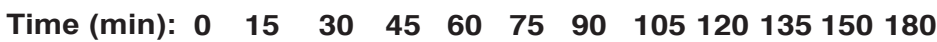

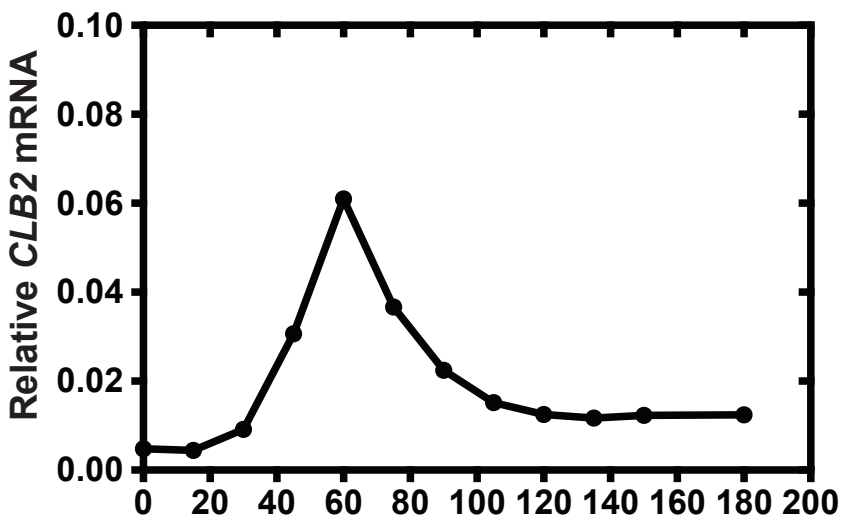

Time after CDC20 shut off ( $\mathrm{min})$
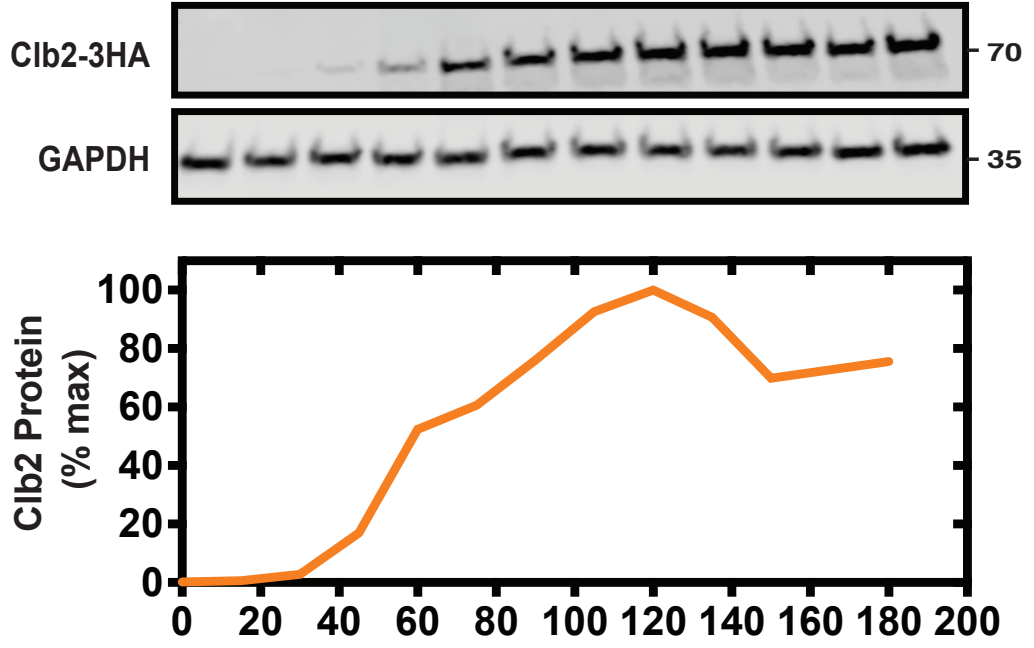

Time after G1 Release into Noc (min)

e

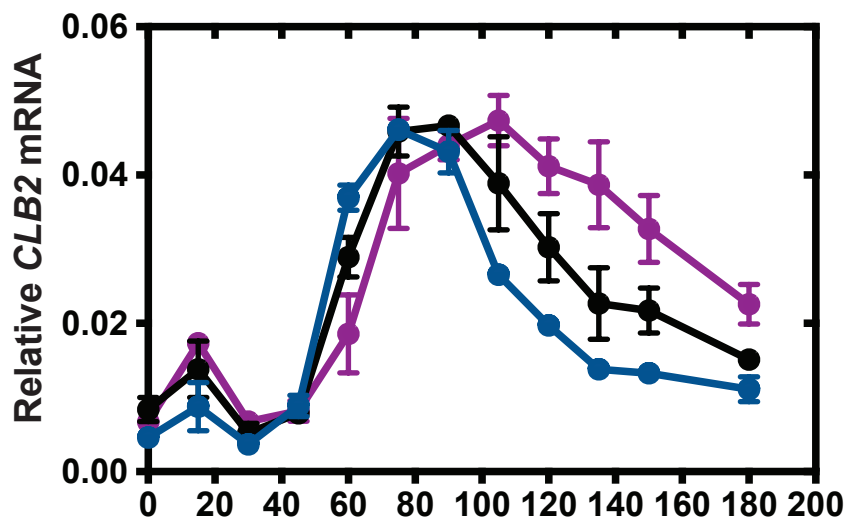

Time after $\mathrm{G} 1$ release into Noc ( $\mathrm{min})$

- Untreated

- 50 nM 1-NM-PP1

100 nM 1-NM-PP1

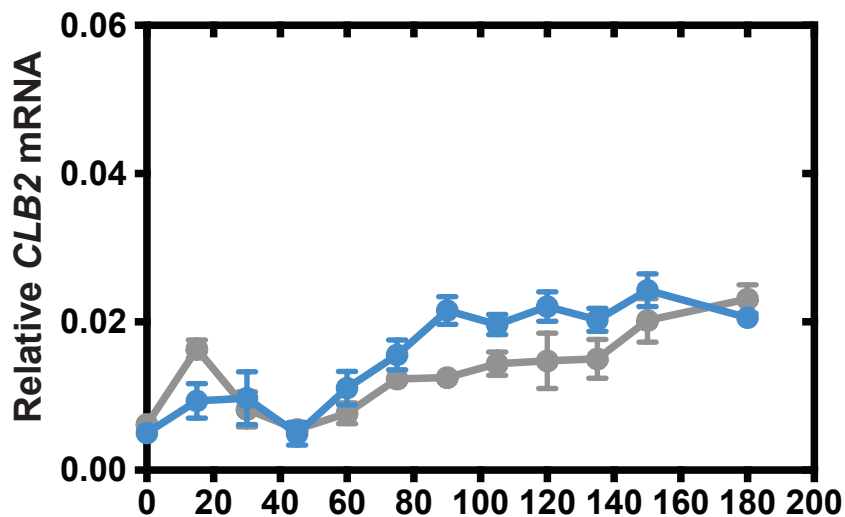

Time after $\mathbf{G} 1$ release into Noc (min)

250 nM 1-NM-PP1

500 nM 1-NM-PP1 
bioRxiv preprint doi: https://doi.org/10.1101/2021.02.14.431158; this version posted Februar/ 14, 2021. The copyright holder for this preprint (which was not certified by peer review) is the author/funder, who has granted bioRxiv a license to display the preprint in perpetuity. It is made

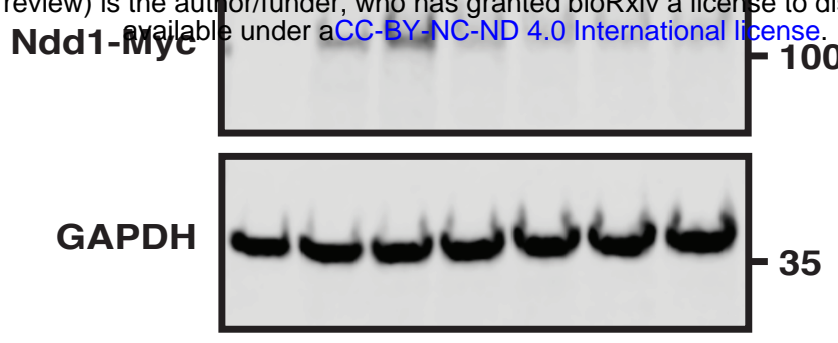

b

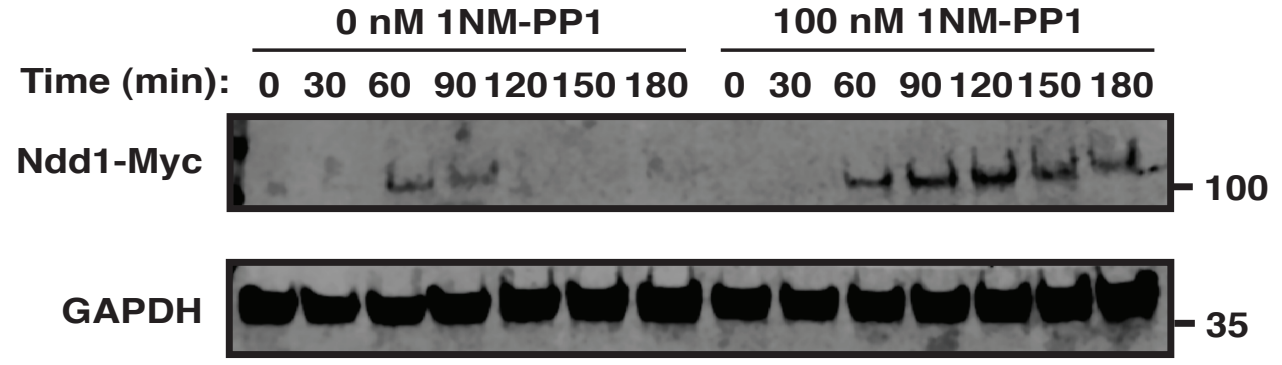

C $\rightarrow 0$ nM 1NM-PP1

60 min post $\mathrm{G} 1$

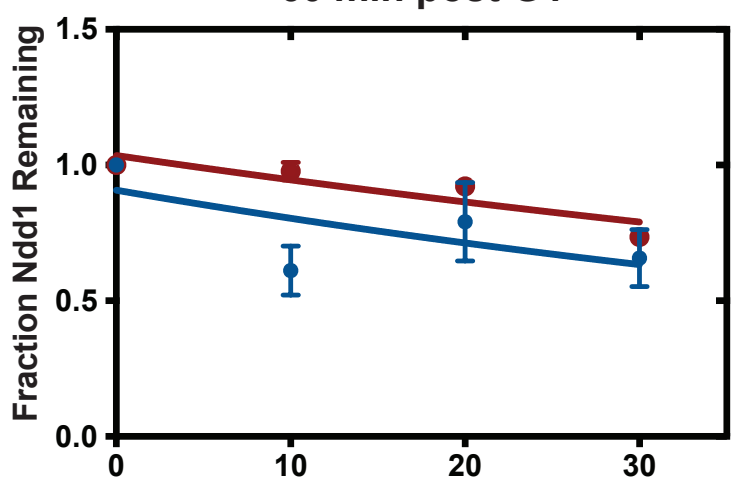

Time after $\mathrm{CHX}$ addition (min)

150 min post $\mathrm{G} 1$

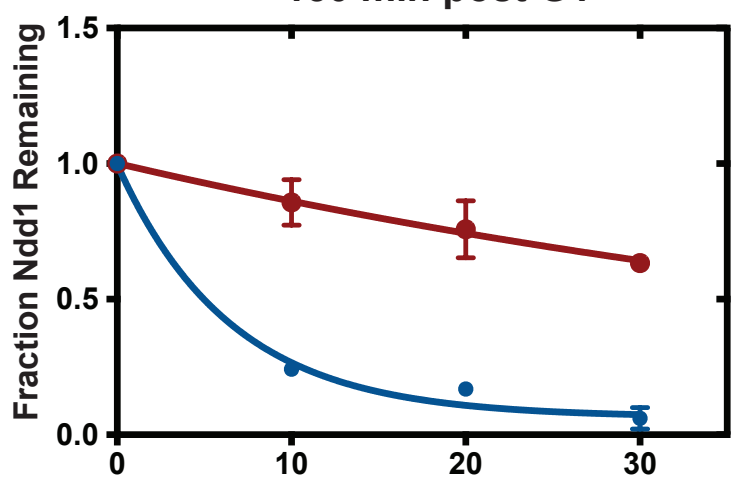

Time after $\mathrm{CHX}$ addition (min) $\rightarrow 500$ nM 1NM-PP1
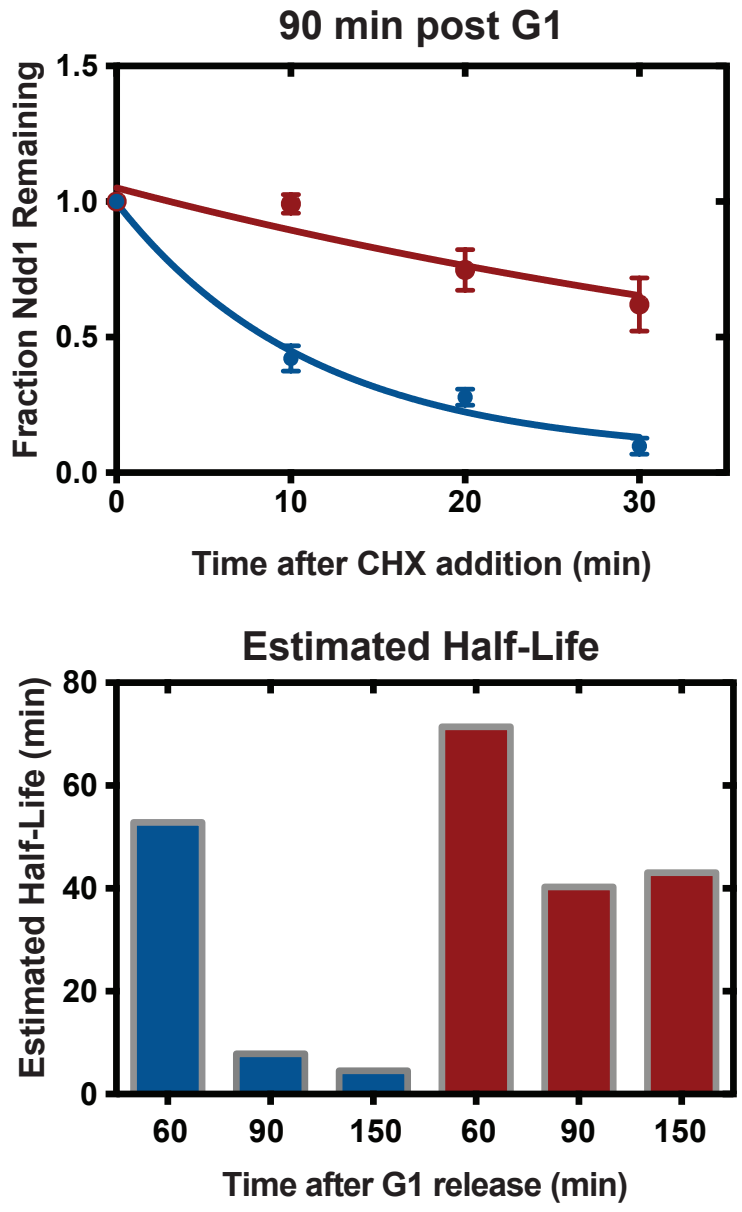

d

CHX (min): $\frac{\text { WT }}{0102030} \quad \frac{\Delta C L B 2}{0102030} \quad \frac{\Delta C L B 3}{0102030}$

Ndd1-Myc

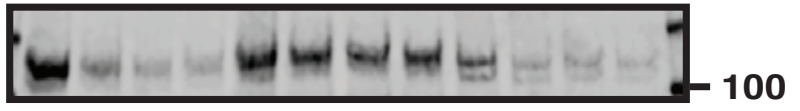


Figure 3

a

bioRxiv preprint doi: https://doi.org/10.1101/2021.02.14.431158; this version posted February 14, 2021. The copyright holder for this preprint (which was not certified by peer review) is the author/funder, who has granted bioRxiv a license to display,the preprint in perpetuity. It is made available under aCC-BY-NC-ND 4.0 International license.
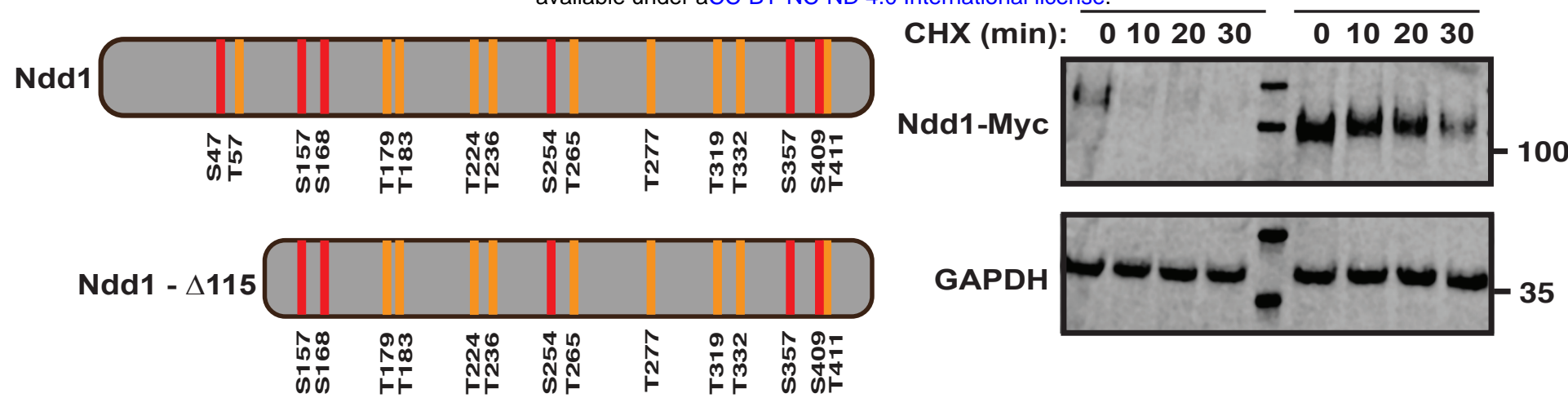

b
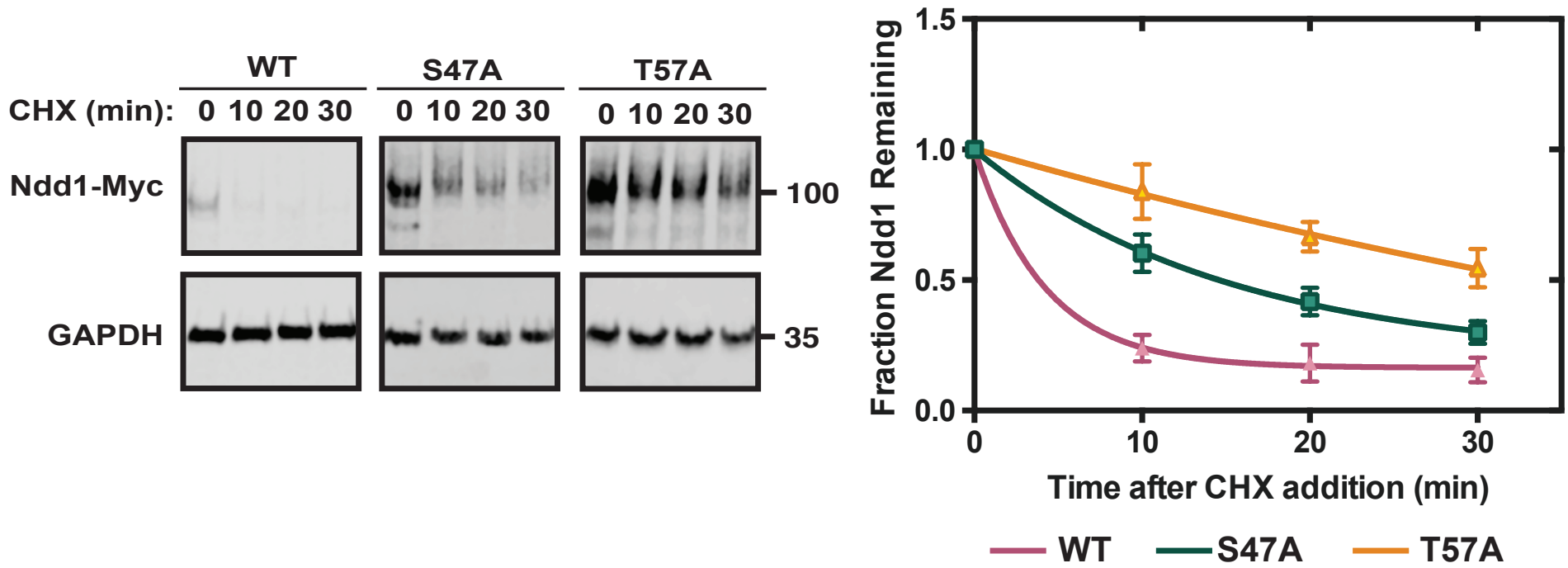

C
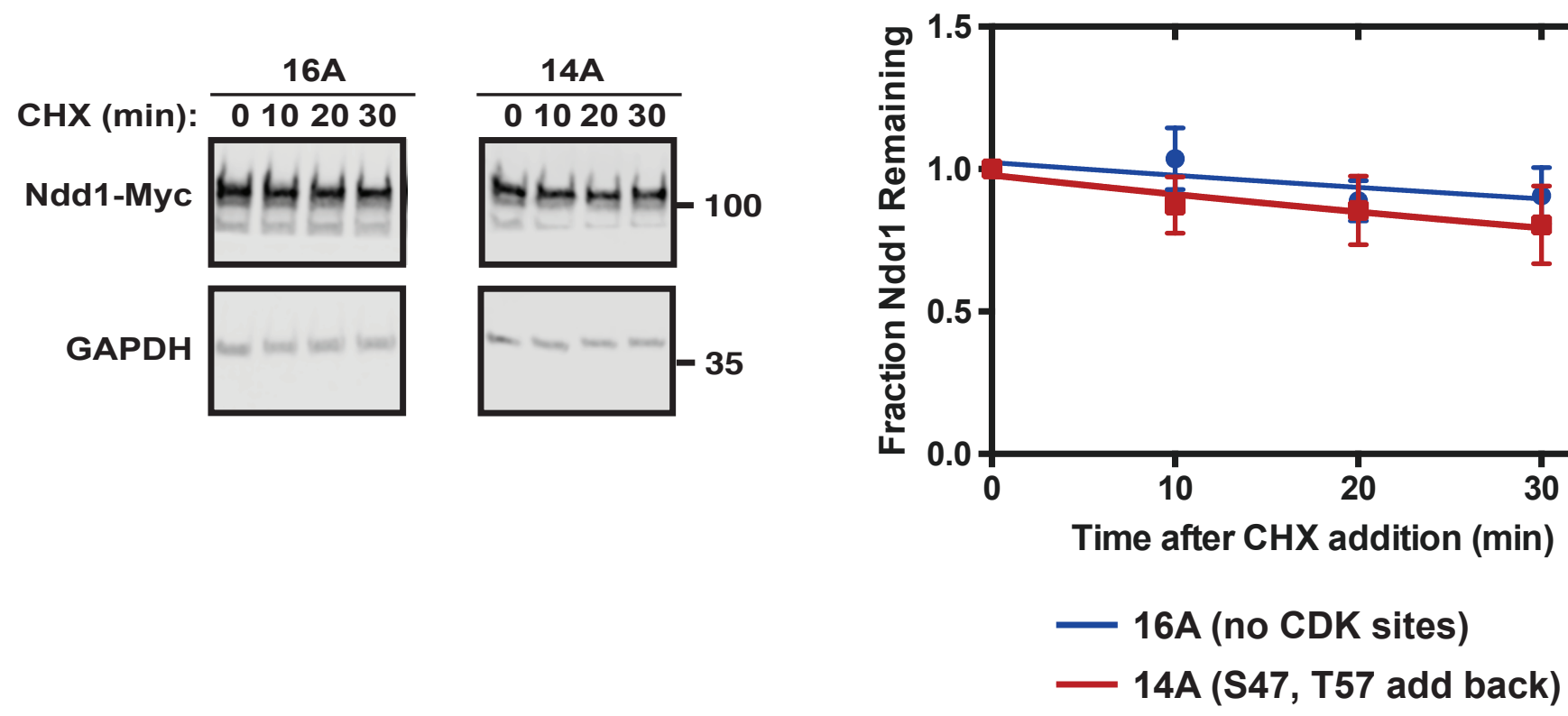
Figure 4

bioRxiv preprint doi: https://doi.org/10.1101/2021.02.14.431158; this version posted February 14, 2021. The copyright holder for this preprint (which was not certified by peer review) is the author/funder, who has granted bioRxiv a license to display the preprint in perpetuity. It is made available under aCC-BY-NC-ND 4.0 International license.

a

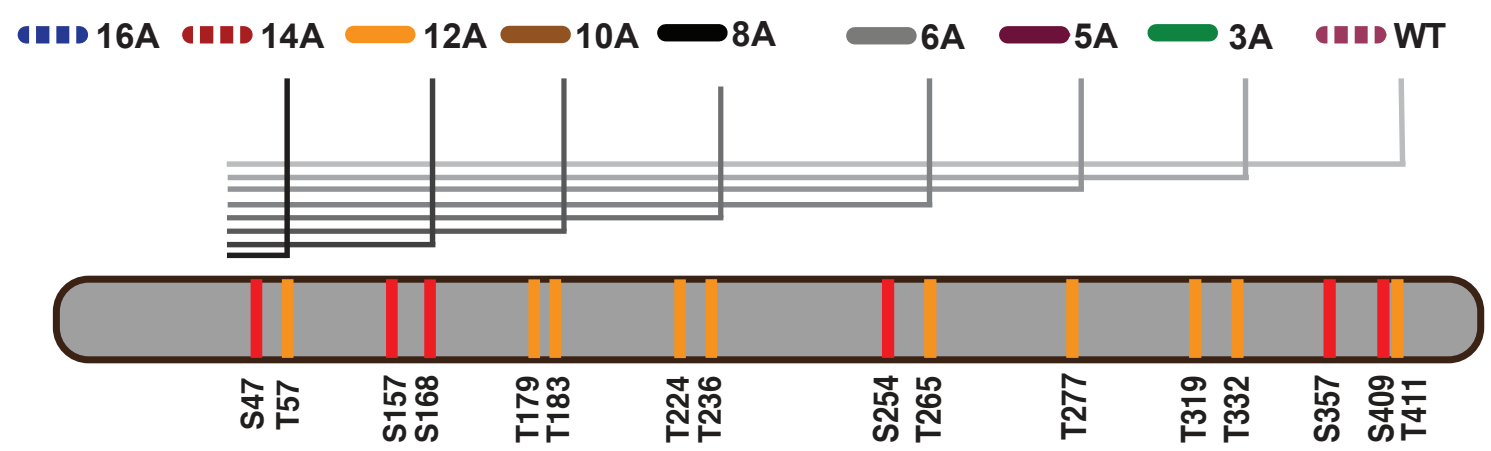

b

$16 \mathrm{~A}$ to $8 \mathrm{~A}$

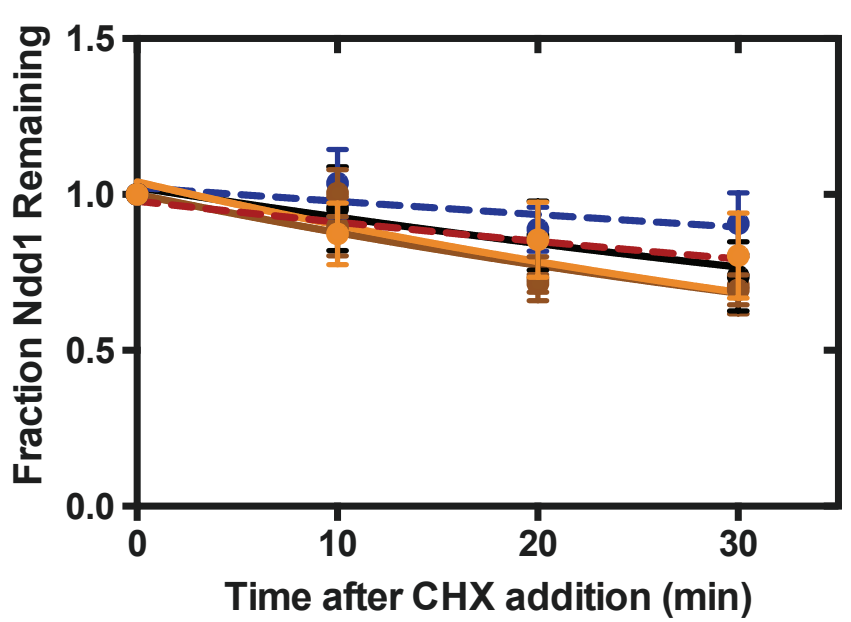

C

\section{A to WT}

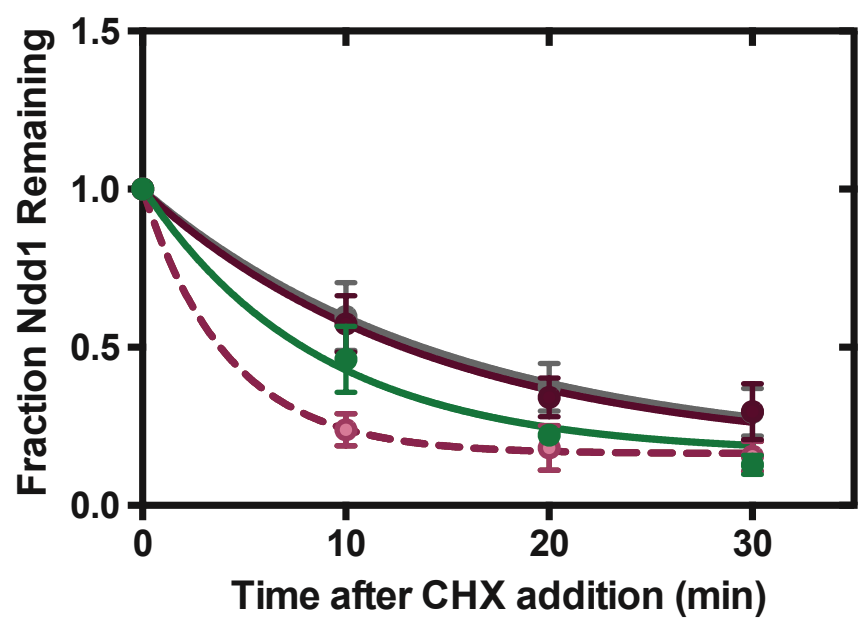


a

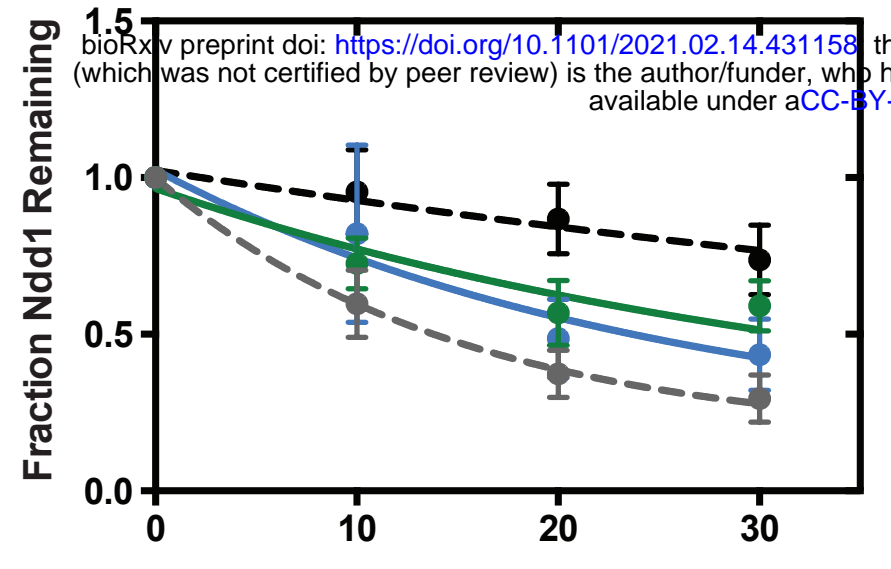

Time after $\mathrm{CHX}$ addition (min)

$$
\begin{aligned}
& -8 A \quad-6 A+5254 A \\
& --6 A-6 A+T 265 A
\end{aligned}
$$

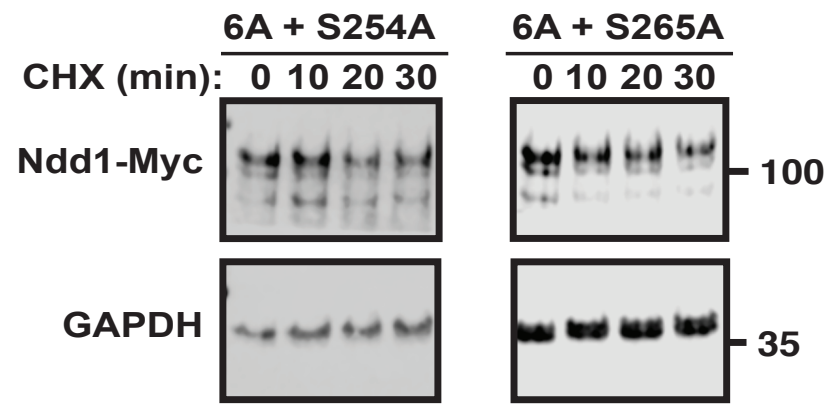

C

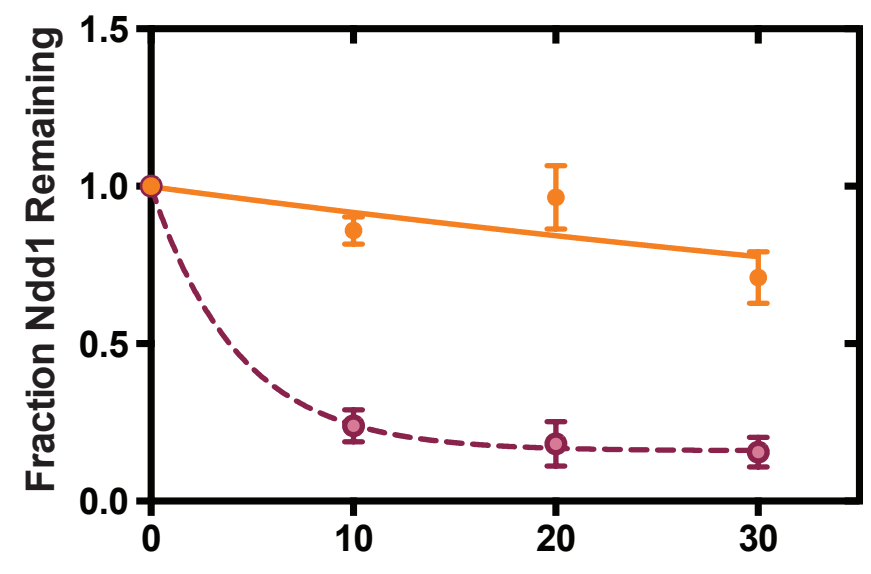

Time after $\mathrm{CHX}$ addition (min)

- Minimal CDK site mutant (S47, T57, S254, T265)

- WT

Minimal CDK site $\begin{array}{ll}\text { CHX (min): } & \frac{\text { mutant }}{0102030} \\ \text { Ndd1-Myc } & \end{array}$

GAPDH

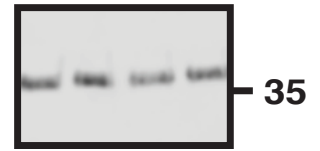

b

ongosted Hebruary 14, 2021. The copyright holder for this preprint

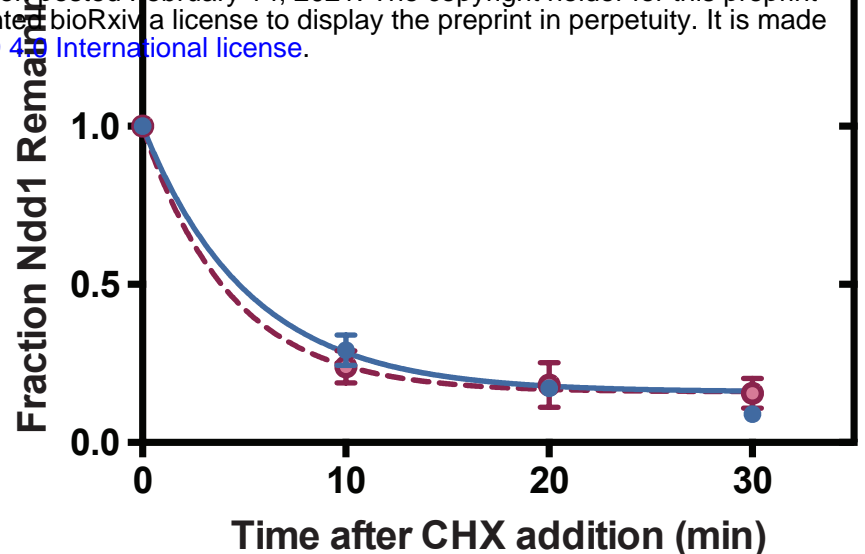

$-254 \mathrm{~A}, 265 \mathrm{~A} \quad--\mathrm{WT}$

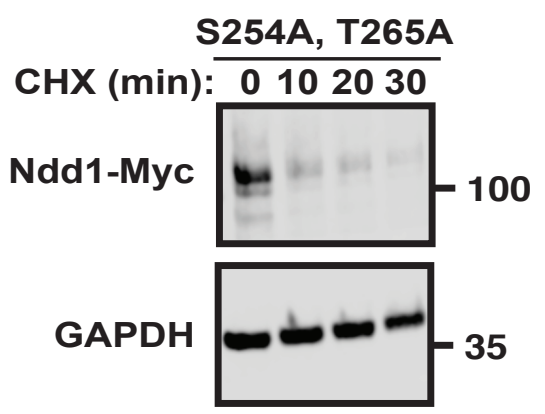

d

Ndd1 - S47A, S157A, S168A, T179A, T183A, T224A, T236A (Ndd1-C1)
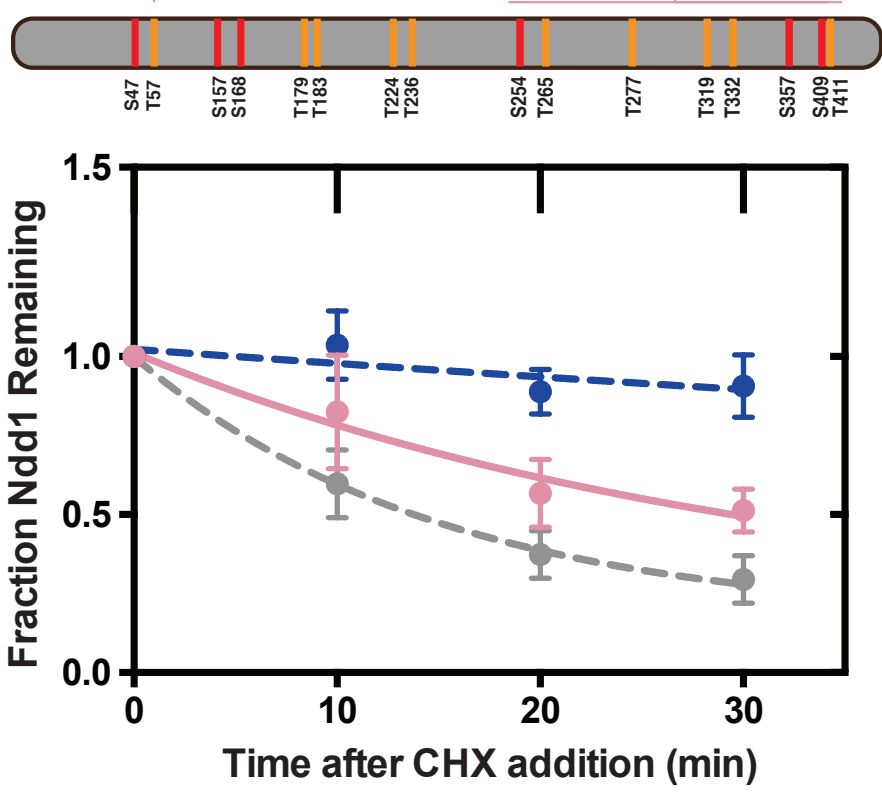

-- Ndd1-16A -- Ndd1-6A $=$ Ndd1-C1

CHX (min): $\frac{\text { Ndd1-C1 }}{0102030}$

Ndd1-Myc

GAPDH 
bioRxiv preprint doi: https://doi.org/10.1101/2021.02.14.431158; this version posted February 14, 2021. The copyright holder for this preprint

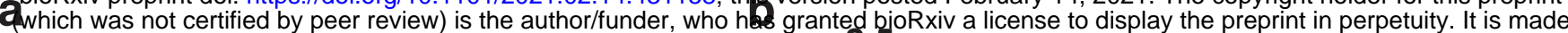

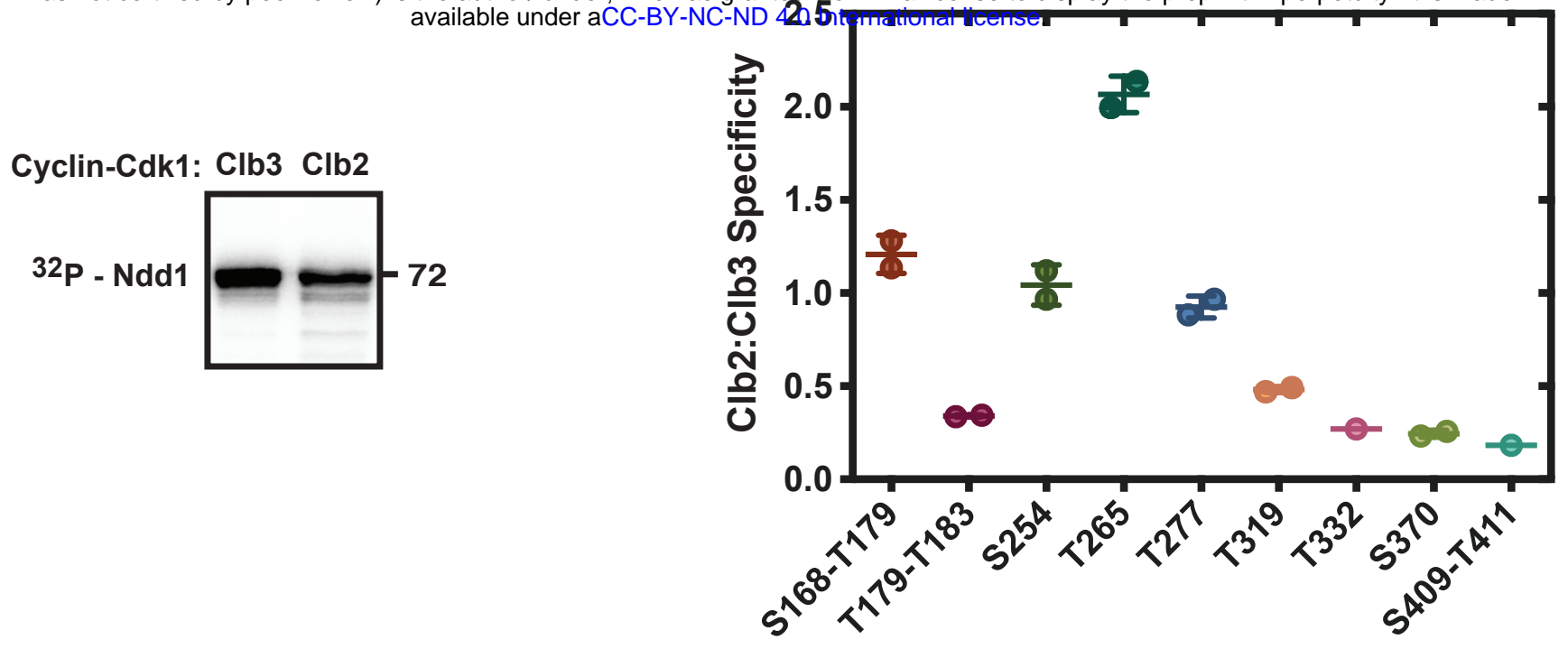

Sites Phosphorylated

C

Ndd1: WT WT 16A 15A

[Clb2-Cdk1]: Low High High High

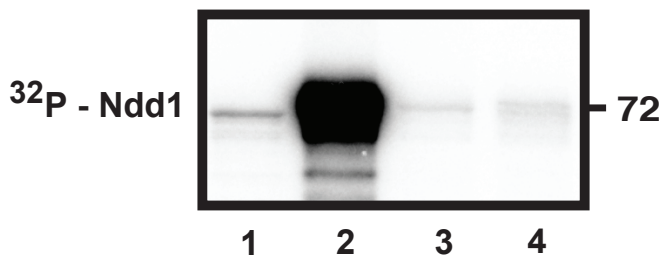

d

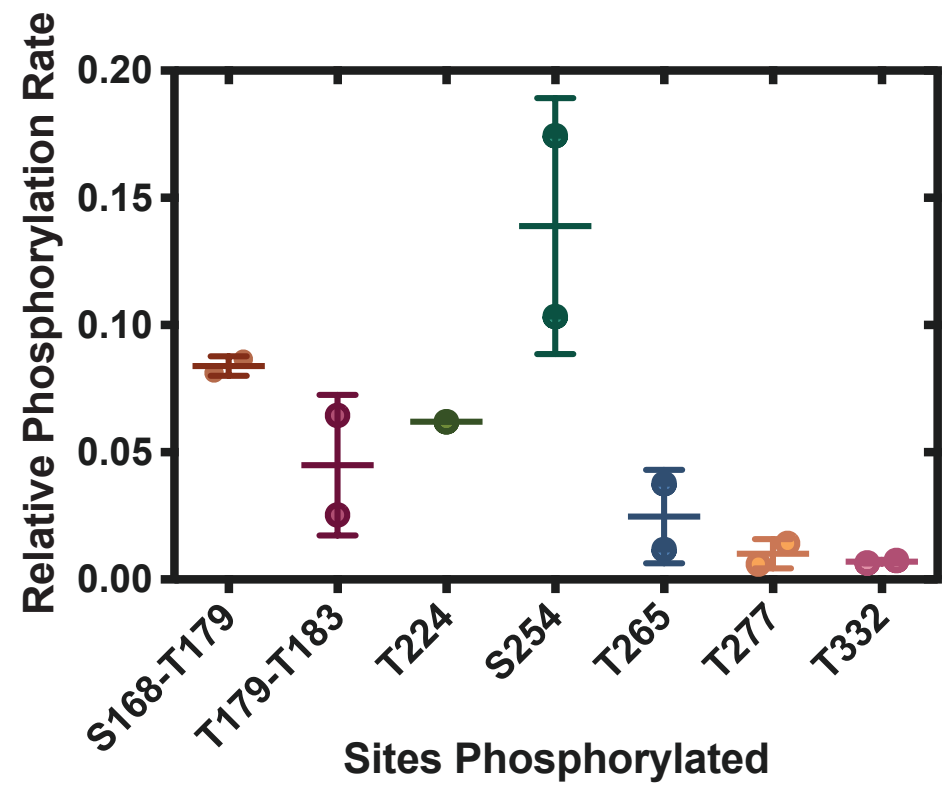

e

$\mathbf{f}$

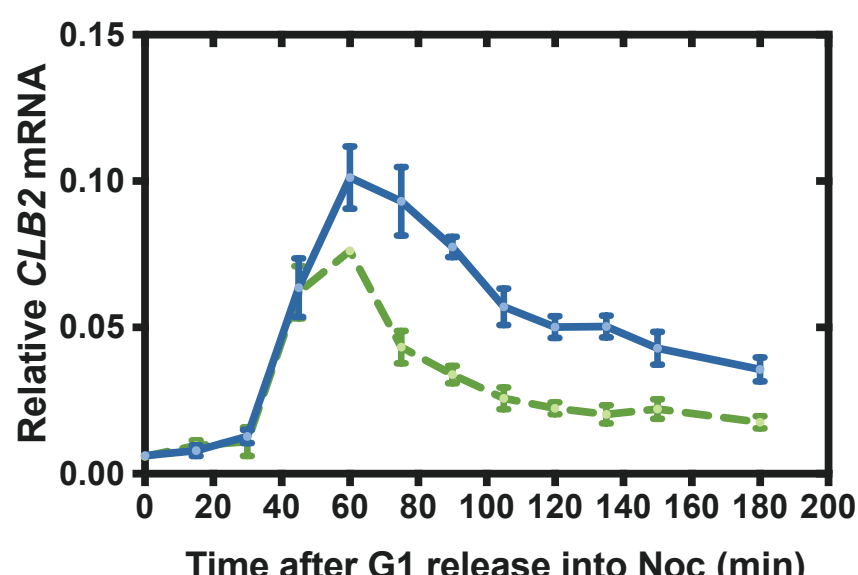

Time after $\mathrm{G} 1$ release into Noc ( $\mathrm{min})$

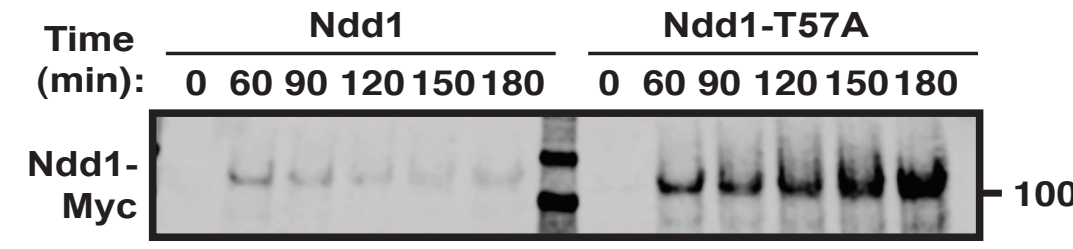

GAPDH

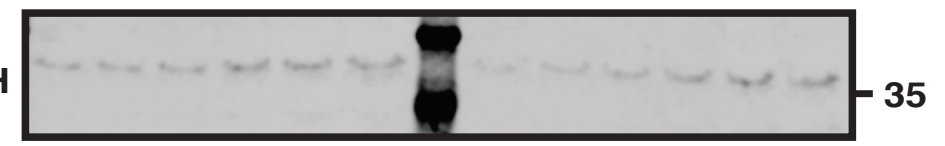

$$
- \text { WT } \quad \text { T57A }
$$




\section{Supplementary Figures - Asfaha et al.}
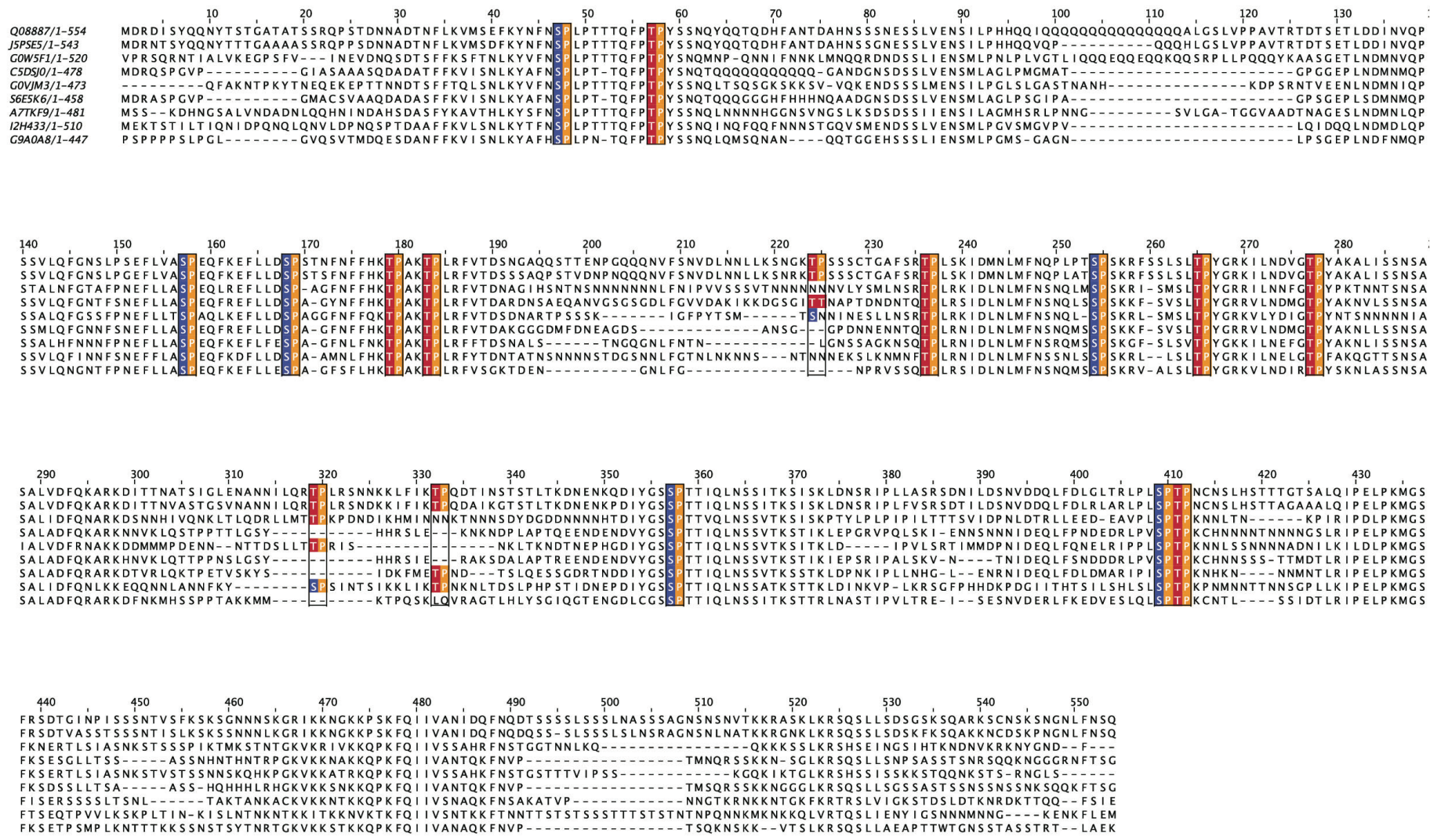

\section{Supplementary Figure 1. Sequence alignment of Ndd1 orthologs from multiple yeast species}

The Ndd1 primary sequence from Saccharomyces cerevisiae was aligned to other budding yeasts using ProViz (http://slim.icr.ac.uk/proviz/). Ndd1 contains multiple sets of predicted CDK sites (highlighted), spaced 10-12 resides apart. The Ndd1 sequences in the alignment are from the following species (Uniprac ID): Saccharomyces cerevisiae (Q08887), Saccharomyces kudriavzevii (J5PSE5), Naumovozyma dairenensis (G0W5F1), Zygosaccharomyces rouxii (C5DSJ0), Naumovozyma castellii (G0VJM3), Zygosaccharomyces bailii (S6E5K6), Vanderwaltozyma polyspora (A7TKF9), Tetrapisispora blattae (I2H433), Torulaspora delbrueckii (G9A0A8) 


\begin{abstract}
MDRDISYQQN YTSTGATATS SRQPSTDNNA DTNFLKVMSE FKYNFNSPLP
TTTQFPTPYS SNQYQQTQDH FANTDAHNSS SNESSLVENS ILPHHQQIQQ

QQQQQQQQQQ QQQALGSLVP PAVTRTDTSE TLDDINVQPS SVLQFGNSLP

SEFLVASPEQ FKEFLLDSPS TNFNFFHKTP AKTPLRFVTD SNGAQQSTTE

NPGQQQNVFS NVDLNNLLKS NGKTPSSSCT GAFSRTPLSK IDMNLMFNQP

LPTSPSKRFS SLSLTPYGRK ILNDVGTPYA KALISSNSAL VDFQKARKDI

TTNATSIGLE NANNILQRTP LRSNNKKLFI KTPQDTINST STLTKDNENK

QDIYGSSPTT IQLNSSITKS ISKLDNSRIP LLASRSDNIL DSNVDDQLFD

LGLTRLPLSP TPNCNSLHST TTGTSALQIP ELPKMGSERS DTGINPISSS

NTVSFKSKSG NNNSKGRIKK NGKKPSKFQI IVANIDQFNQ DTSSSSLSSS

LNASSSAGNS NSNVTKKRAS KLKRSQSLLS DSGSKSQARK SCNSKSNGNL

FNSQ
\end{abstract}

\title{
Supplementary Figure 2. Ndd1 mass spectrometry sequence coverage
}

Peptides containing sequences detected by mass spectrometry in Figure 6 are indicated in bold red text. Sequences that were not detected are indicated in black text. 


\section{Supplementary Table 1. Yeast strains used in this work}

\begin{tabular}{|c|c|c|}
\hline Strain & Genotype & Figure \\
\hline DOM0090 & Wild-type W303 (AFS92, A. Straight): MATa, bar1::HisG & $1 a, b, 6 f$ \\
\hline yJA001 & cdc20::pGAL & $1 \mathrm{c}$ \\
\hline yJA002 & clb2::CLB2-3XHA & $1 d$ \\
\hline DOM0030 & cdc28::CDC28-AS1 & $1 e, f$ \\
\hline yJA003 & ndd1::NDD1-9xMYC-HYGR & $2 a, 6 f$ \\
\hline yJA004 & cdc28::CDC28-AS1 ndd1::NDD1-9xMYC-HYGR & $2 b, c$ \\
\hline yJA005 & trp1::pGAL-NDD1-9XMYC-TRP1 & $2 d, 3 a, 3 b, 4 c, 5 b, 5 c$ \\
\hline yJA006 & clb2::KANMX trp1::pGAL-NDD1-9XMYC-TRP1 & $2 d$ \\
\hline yJA007 & clb3::KANMX trp1::pGAL-NDD1-9XMYC-TRP1 & $2 d$ \\
\hline yJA008 & trp1::pGAL- $\Delta 1-115-N D D 1-9 X M Y C-T R P 1$ & $3 a$ \\
\hline yJA009 & trp1::pGAL-NDD1-S47A-9XMYC-TRP1 & $3 b$ \\
\hline yJA010 & trp1::pGAL-NDD1-T57A-9XMYC-TRP1 & $3 b$ \\
\hline yJA011 & $\begin{array}{l}\text { trp1::pGAL-NDD1-S47A-T57A-S157A-S168A-T179A-T183A-T224A- } \\
\text { T236A-S254A-T265A-T277A-T319A-T332A-S357A-S409A-T411A- } \\
\text { 9XMYC-TRP1 }\end{array}$ & $3 c, 4 b, 5 d$ \\
\hline yJA012 & $\begin{array}{l}\text { trp1::pGAL-NDD1-S157A-S168A-T179A-T183A-T224A-T236A-S254A- } \\
\text { T265A-T277A-T319A-T332A-S357A-S409A-T411A-9XMYC-TRP1 }\end{array}$ & $3 c, 4 b$ \\
\hline yJA013 & $\begin{array}{l}\text { trp1::pGAL-NDD1-T179A-T183A-T224A-T236A-S254A-T265A-T277A- } \\
\text { T319A-T332A-S357A-S409A-T411A-9XMYC-TRP1 }\end{array}$ & $4 \mathrm{~b}$ \\
\hline yJA014 & $\begin{array}{l}\text { trp1::pGAL-NDD1-T224A-T236A-S254A-T265A-T277A-T319A-T332A- } \\
\text { S357A-S409A-T411A-9XMYC-TRP1 }\end{array}$ & $4 \mathrm{~b}$ \\
\hline yJA015 & $\begin{array}{l}\text { trp1::pGAL-NDD1-S254A-T265A-T277A-T319A-T332A-S357A-S409A- } \\
\text { T411A-9XMYC-TRP1 }\end{array}$ & $4 b, 5 a$ \\
\hline yJA016 & $\begin{array}{l}\text { trp1::pGAL-NDD1-T277A-T319A-T332A-S357A-S409A-T411A-9XMYC- } \\
\text { TRP1 }\end{array}$ & $4 c, 5 a, 5 d$ \\
\hline yJA017 & trp1::pGAL-NDD1-T319A-T332A-S357A-S409A-T411A-9XMYC-TRP1 & $4 \mathrm{c}$ \\
\hline yJA018 & trp1::pGAL-NDD1-S357A-S409A-T411A-9XMYC-TRP1 & $4 c$ \\
\hline yJA019 & $\begin{array}{l}\text { trp1::pGAL-NDD1-T265A-T277A-T319A-T332A-S357A-S409A-T411A- } \\
\text { 9XMYC-TRP1 }\end{array}$ & $5 a$ \\
\hline yJA020 & $\begin{array}{l}\text { trp1::pGAL-NDD1-S254A-T277A-T319A-T332A-S357A-S409A-T411A- } \\
\text { 9XMYC-TRP1 }\end{array}$ & $5 a$ \\
\hline yJA021 & trp1::pGAL-NDD1-S254A, T265A-9XMYC-TRP1 & $5 b$ \\
\hline yJA022 & $\begin{array}{l}\text { trp1::pGAL-NDD1-S157A-S168A-T179A-T183A-T224A-T236A-T277A- } \\
\text { T319A-T332A-S357A-S409A-T411A-9XMYC-TRP1 }\end{array}$ & $5 c$ \\
\hline yJA023 & $\begin{array}{l}\text { trp1::pGAL-NDD1-S47A-S157A-S168A-T179A-T183A-T224A-T236A- } \\
\text { 9XMYC-TRP1 }\end{array}$ & $5 d$ \\
\hline yJA024 & trp1::pNDD1-NDD1-T57A & $6 e$ \\
\hline yJA025 & ndd1::HYGR trp1::pNDD1-NDD1-T57A & $6 f$ \\
\hline
\end{tabular}

